\title{
Constitutive AKT Activity Predisposes Lung Fibrosis by Regulating Macrophage, Myofibroblast and Fibrocyte Recruitment and Changes in Autophagy
}

\author{
Duaa Dakhlallah",2\#, Yijie Wang1\#, Tierra A. Bobo',2, Emily Ellis², Xiaokui Mo3 , Melissa G. Piper ${ }^{1}$, \\ Timothy D. Eubank ${ }^{1,2,4}$, Clay B. Marsh1,2* \\ ${ }^{1}$ Division of Pulmonary, Allergy, Critical Care \& Sleep Medicine, Davis Heart and Lung Research Institute, The Ohio State \\ University, Columbus, OH, USA \\ ${ }^{2}$ Robert C. Byrd Health Sciences Center, West Virginia University, Morgantown, WV, USA \\ ${ }^{3}$ The Center for Biostatistics, The Ohio State University, Columbus, OH, USA \\ ${ }^{4}$ Department of Microbiology, Immunology and Cell Biology, West Virginia University, Morgantown, WV, USA \\ Email: *cbmarsh@hsc.wvu.edu
}

How to cite this paper: Dakhlallah, D., Wang, Y.J., Bobo, T.A., Ellis, E., Mo, X.K., Piper, M.G., Eubank, T.D. and Marsh, C.B. (2019) Constitutive AKT Activity Predisposes Lung Fibrosis by Regulating Macrophage, Myofibroblast and Fibrocyte Recruitment and Changes in Autophagy. Advances in Bioscience and Biotechnology, 10, 346-373.

https://doi.org/10.4236/abb.2019.1010027

Received: September 6, 2019

Accepted: October 27, 2019

Published: October 30, 2019

Copyright $\odot 2019$ by author(s) and Scientific Research Publishing Inc. This work is licensed under the Creative Commons Attribution International License (CC BY 4.0).

http://creativecommons.org/licenses/by/4.0/

\begin{abstract}
The etiology and pathogenesis of pulmonary fibrosis is poorly understood. We and others reported that M-CSF/CSF-1, M-CSF-R and downstream AKT activation plays an important role in lung fibrosis in mice models and in IPF patients. To understand potential molecular pathways used by M-CSF-R activation to direct lung fibrosis, we used a novel transgenic mouse model that expresses a constitutively-active form of AKT, myristoylated AKT (Myr-Akt), driven by the $c$-fms (M-CSF-R) promoter. We were particularly interested in the basal immune state of the lungs of these Myr-Akt mice to assess M-CSF-R-related priming for lung fibrosis. In support of a priming effect, macrophages isolated from the lungs of unchallenged Myr-Akt mice displayed an M2-tropism, enhanced co-expression of M-CSF-R and $\alpha$-SMA, reduced autophagy reflected by reduced expression of the key autophagy genes Beclin-1, MAP1-Lc3a(Lc3a), and MAP1-Lc3b(Lc3b), and increased $p 62 / S T S Q M 1$ expression compared with littermate WT mice. Furthermore, Myr-Akt mice had more basal circulating fibrocytes than WT mice. Lastly, upon bleomycin challenge, Myr-Akt mice showed enhanced collagen deposition, increased F4/80+ and CD45+ cells, reduced autophagy genes Beclin-1, Lc3a, and Lc3b expression, and a shorter life-span than WT littermates. These data provide support that M-CSF-R/AKT activation may have a priming effect which can predispose lung tissue to pulmonary fibrosis.
\end{abstract}




\section{Keywords}

Pulmonary Fibrosis, AKT, CSF1, M-CSF Receptor, Macrophage, Myofibroblast, Fibrocytes, Autophagy

\section{Introduction}

Idiopathic pulmonary fibrosis (IPF) is a progressive respiratory disease with a 5 -year mortality rate of 50\% - 70\% [1]. IPF is characterized pathologically by destruction of lung architecture, fibrotic remodeling, accumulation of myofibroblasts, and deposition of extracellular matrix (ECM) [2]. To date, the etiology and pathogenesis of IPF remains unclear.

The serine/threonine protein kinase, AKT, is downstream of many receptor tyrosine kinases and plays an important role in cell survival, differentiation, and cellular activation [3]. In macrophages, activation of the M-CSF receptor (M-CSF-R) induces stimulation of phosphoinositide 3-kinase (PI3K) leading to AKT recruitment to the cell membrane and subsequent Thr308 and Ser437 phosphorylation to activate AKT [4]. In fibroblasts, activated AKT regulates collagen I and III production [5] and contributes to human hepatic fibrosis [6] and bleomycin-induced lung fibrosis in mice [7] [8]. Importantly, PI3K/AKT activity is enhanced in IPF fibroblasts due to reduced levels of PTEN phosphatase function [9]. Moreover, PI3K/AKT inhibitors such as PX-866 are potential therapeutic agents for IPF [7] [10]. Thus, understanding the role of activated AKT is important in IPF.

Macrophages express several fibrotic mediators and play an important role in wound healing and organ fibrosis [11] [12] [13] [14]. There are two main types of macrophages with disparate inflammatory functions: classically-activated "killing" macrophages (M1 phenotype) activated by microbial agents to express pro-inflammatory cytokines like TNF- $\alpha$, IL-18, IL-12, IL-6, IL-1 and inducible nitric oxide synthase (iNOS) that enhance type I helper $\mathrm{T}$ cells to proliferate [15] [16] [17], and alternatively-activated, or M2 "repair" macrophages that express relatively higher levels of the anti-inflammatory cytokines IL-10, mannose receptor-1 (MRC)-1, YM1, and arginase-1 (ARG1) and Th2-type cytokines. M2 macrophages exhibit pro-fibrotic, pro-angiogenic, and anti-inflammatory activities [15] [16] [18]. Several studies suggest M2 macrophages contribute to fibrotic lung diseases like IPF [11] [19] [20]. Thus, understanding the molecular regulation of M2 macrophages may be important in pulmonary fibrosis.

Another critical effector cell type in IPF is myofibroblasts, which secrete excessive extracellular matrix proteins like collagen I and III that contribute to fibrosis [19] [21]. Myofibroblasts are identified by their expression of $\alpha$-smooth muscle actin ( $\alpha$-SMA) and can differentiate from resident lung fibroblasts, epithelial-mesenchymal cells, or circulating fibrocytes [21] [22]. Interestingly, $\mathrm{PI} 3 \mathrm{~K} / \mathrm{AKT}$ is involved in TGF $\beta$-induced myofibroblast proliferation and diffe- 
rentiation in IPF [23]. Thus, myofibroblast function is critical in IPF.

In addition to M2 macrophages and myofibroblasts, circulating fibrocytes are also associated with pulmonary fibrosis [24]. Fibrocytes are bone marrow-derived mesenchymal cells and express leukocyte, hematopoietic cell and fibroblast markers [25]. Fibrocytes express chemokine receptors and adhesion molecules and can traffic to fibrotic regions [24]. Furthermore, fibrocytes can deposit the ECM proteins collagen I and III [26] and can also differentiate into fibroblasts and myofibroblasts, which further contribute to ECM deposition [27]. Therefore, circulating fibrocytes can contribute to the pathogenesis of pulmonary fibrosis [24] [28].

Reduced cellular homeostatic processes like autophagy are also found in the lungs of patients with IPF [29] [30] [31]. Autophagy is an evolutionarily-conserved lysosome-dependent cellular pathway responsible for the processing of unhealthy organelles and cellular waste to maintain cellular homeostasis during cellular stress [32]. Successful autophagy results in the delivery of proteins and/or organelles into double-membrane autophagosomes for lysosomal degradation [32] [33] and cellular homeostasis. Interestingly, autophagy activity correlates with organismal longevity [34] and interruption of autophagy is associated with cellular aging. Several studies report reduced autophagic vesicle formation (autophagosomes) and activity in human IPF lung tissue as measured by reduced LC3A/B-II (microtubule associated protein 1 light chain 3) and increased p62 (chaperone protein to autophagosome) [29] [30] [31]. BECLIN-1, a major regulator of autophagy, is decreased in IPF lung fibroblasts. Furthermore, BECLIN-1 also binds to BCL-2 and decreases apoptosis in these fibroblasts [31] [35]. As an autophagy regulator, mammalian target of rapamycin, or mTOR, kinase activity is elevated in IPF lung fibroblasts [36] via enhanced AKT activation and PTEN suppression [37]. Thus, pathways regulating autophagy in IPF likely contribute to lung fibrosis.

Previous data generated from our laboratory revealed that CSF1 contributes to pulmonary fibrosis in mice as CSF1-/-mice showed less fibrosis in response to bleomycin challenge [38]. However, the specific mechanism(s) of M-CSF-R-mediated signaling and regulatory pathways connecting CSF1 and M-CSF-R signaling to specific target cell populations is unclear. Current knowledge in the field is that AKT activation, M2 macrophage polarity, increased myofibroblast and fibrocytes numbers, and reduced autophagy are observed in the lungs of patients with IPF. Here, we extend our previous study by using a murine model, $c$-fms-directed Myr-Akt expression, to examine the role of constitutive AKT activation driven by the M-CSF-R promoter and identify potential priming of the lungs to fibrosis. With this murine model, we study a potential causal relationship between M-CSF-R-directed AKT activation and cellular phenotype to drive fibrosis. We observed that lungs from Myr-Akt mice had increased expression of genes representative of M2 macrophages, increased $\alpha$-SMA positive cells, increased number of fibrocytes and reduced autophagy-related transcripts compared to WT mice. These data suggest M-CSF-R/AKT activation may predispose 
a pulmonary fibrotic phenotype.

\section{Materials and Methods}

\subsection{Ethics Statement}

This study was carried out in strict accordance with the recommendations in the Guide for the Care and Use of Laboratory Animals of the National Institutes of Health. The animal protocols were approved by the Institutional Laboratory Animal Care and Use Committee (ILACUC) of The Ohio State University (protocols: 2011A00000077 and 2009A0124-R1).

\subsection{Reagents}

Endotoxin-free RPMI 1640 (15-040) and DPBS (21-031) were purchased from Corning Cellgro. Endotoxin-free (certified less than $0.06 \mathrm{EU} / \mathrm{ml}$ ) fetal bovine serum (FBS, S11150) was obtained from Atlanta Biologicals. Recombinant mouse CSF1 (416-ML) was purchased from R\&D Systems. Anti-mouse CD45 antibody (clone 30-F11, 103112), Alexa488 anti-mouse F4/80 (clone BM8, 123120), PerCP/Cy5.5 anti-mouse CXCR4 (clone L276F12, 146510), and APC anti-mouse CD115 (CSF-1R/M-CSF-R) (clone AFS98, 135510) were purchased from Biolegend. Anti-rabbit $\alpha \mathrm{SMA}$ (ab5694) and anti-mouse Collagen I (clone COL-1, ab6308) were obtained from Abcam. LC3B (D11) XP Rabbit mAb (3868) and Alexa Fluor 488 conjugate secondary antibody was purchased from Cell Signaling Technology. FITC anti-mouse $\alpha$-SMA antibody (clone 1A4, F3777) was purchased from Sigma-Aldrich. Cytofix/Cytoperm solution (554714) was purchased from BD Biosciences. Primers and SYBR Green Master Mix (4385610) for quantitative real-time polymerase chain reaction (qRT-PCR) were purchased from Thermofisher Scientific unless indicated otherwise. Bleomycin was obtained from Sicor Pharmaceuticals (Irvine, CA).

\subsection{Animal Model}

Constitutively-active myristoylated Myr-Akt transgenic mice were generated by Dr. Michael Ostrowski (OSU, Columbus, $\mathrm{OH}$ ) as described previously [39]. Briefly, a $c$-fms-Myr-Akt plasmid was used to generate the mouse expressing a membrane-bound form of Akt1 in mononuclear phagocytes. The myristoylation tag on Akt facilitates membrane binding resulting in constitutive activation (Supplemental Figure 1(a)). 6 - 8 week old male and female mice were sacrificed by cardiac puncture after isoflurane inhalation and lungs perfused with 25 $35 \mathrm{~mL}$ DPBS. Tissues were fixed in 10\% formalin for histologic and immunohistochemical analysis, snap-frozen in liquid nitrogen for RNA analysis, or digested immediately to obtain a single cell suspension for flow cytometry analysis. Bone marrow-derived macrophages (BMM) were obtained from Myr-Akt mice or WT littermate mice by culture in RPMI 1640 media supplemented with 10\% FBS, 1\% PSA (Penicillin-Streptomycin Amphotericin), $5 \mu \mathrm{g} / \mathrm{mL}$ polymyxin B, and $20 \mathrm{ng} / \mathrm{ml}$ recombinant murine CSF1 as described [40]. 3 - 5 mice per group 
were used in the experiments as indicated in the Figures and Legends. Bleomycin treatment was administered as described [38] using 6 - 12 week old male mice.

\subsection{Immunohistochemistry (IHC)}

Immunohistochemical processing and staining with hematoxylin and eosin (H\&E), Masson's Trichrome, F4/80 or $\alpha$-SMA staining were done at the Histology Core at OSU Veterinarian School as described [41]. For LC3B staining, mouse lungs were fixed in freshly made $4 \%$ paraformaldehyde for 24 hours and processed and stained at OSU Wexner Medical Center Pathology Core. Ten pictures per section were captured using the Olympus IX50 inverted microscope-equipped Nikon camera (Olympus). The average number of pixels corresponding to specific stains was quantified using Adobe Photoshop CS and histogram analysis (Adobe) as described [41]. Data are presented as percent positive cells per high-power field (HPF) and are expressed as the mean \pm SEM.

\subsection{Single Cell Suspension and Flow Cytometry}

Mouse tissue were harvested and immediately homogenized with the McIlwain Tissue Chopper (Ted Pella, Inc.) to obtain a single-cell suspension as described previously [41]. Briefly, mechanically homogenized tissue was digested in RPMI 1640 medium containing $0.525 \mathrm{mg} / \mathrm{mL}$ collagenase Type 1 (Worthington Biochemical Corporation), $0.02 \mathrm{mg} / \mathrm{mL}$ Deoxyribonuclease I (Sigma), and 10\% FBS in a $37^{\circ} \mathrm{C}$ shaker for 1 hour at $250 \mathrm{rpm}$. The homogenates were passed through a $70 \mu \mathrm{m}$ filter and red blood cells were lysed with RBC Lysis Buffer $(0.034 \mathrm{M}$ aluminum chloride, $0.01 \mathrm{M}$ potassium bicarbonate, $0.1 \mathrm{mM}$ EDTA in $\mathrm{dH}_{2} \mathrm{O}$ ) for 30 sec. Cells were suspended in flow wash buffer ( $1 \%$ BSA and $0.1 \%$ sodium azide in DPBS) and an equal number of cells subjected to flow cytometry. Cells were first incubated with Chrom Pure Mouse IgG (Jackson Immuno Research) to block Fc $\gamma$ receptors. Cells were then stained with extracellular antigen antibody on ice for 30 minutes prior to permeabilization/fixation on ice for 20 minutes and intracellular staining on ice for an additional 30 minutes. Cells were washed and measured with the BD LSR II flow cytometer (BD Biosciences). Data was analyzed using FCS3 Express software (De Novo).

\subsection{Fibrocytes Isolation}

Primary murine fibrocytes were isolated from freshly collected peripheral blood as described [42]. Briefly, blood was collected from cardiac puncture and PBMCs were isolated using Lympholyte-Mammal (Cedarlene). PBMCs were cultured in FibroLife basal media (Lifeline Cell Technology) supplemented with $20 \mathrm{mM}$ HEPES, $2 \times$ NEAA, $2 \mathrm{mM}$ sodium pyruvate, $4 \mathrm{mM}$ glutamine, $100 \mathrm{U} / \mathrm{mL}$ penicillin, $100 \mu \mathrm{g} / \mathrm{ml}$ streptomycin, and $2 \times$ ITS- 3 for 5 days to generate mouse fibrocytes. Cells were counted and an equal number of cells were stained with antibodies specific for CD45 and CXCR4, permeabilized/fixed, and stained for Collagen I. The cells were analyzed by flow cytometry. 


\subsection{Analysis of Lung Inflammation by Pathological Assessment}

A board-certified veterinary pathologist subjectively analyzed in a blinded manner and assessed the lungs for inflammation, the number of macrophages, and the amount of fibrous connective tissue present within the H\&E- and Trichrome-stained slides, and scored them accordingly as previously described [38].

\subsection{RNA Extraction and Quantitative Real-Time PCR}

Total RNA was extracted using TRIzol (Thermofisher Scientific) and $1 \mu \mathrm{g}$ total RNA was used to synthesize cDNA with SuperScript III Reverse Transcriptase (Thermofisher Scientific). Quantitative real-time (qRT)-PCR was performed in duplicate using SYBR Green Master Mix (Applied BioSystems) and an ABI PRIZM 7700 with Sequence Detector v1.7 (Applied Biosystems). Primer sequences for mouse collagen $1 A$, collagen $3 B$, IL-12, iNos, IL10, Ym1, Atg3, Atg5, Beclin-1, $L c 3 a$ and $L c 3 b$ are listed in Supplemental Table 1. Target genes were normalized to the average values of housekeeping genes Cap1, Rpl4 (Thermofisher Scientific) and Gapdh [38] and expressed as relative copy number (RCN) using $2^{(-\Delta \mathrm{Ct})}$.

\subsection{Western Blot}

Single cell suspensions were obtained and lysed in Cell Lysis Buffer with protease inhibitors (Cell Signaling Technology). Protein concentration was determined by the Dc protein assay (Bio-Rad) and equal amounts of protein were separated on $10 \%$ or $4 \%-12 \%$ NuPAGE Novex bis-tris gel (Thermofisher Scientific), transferred to a nitrocellulose membrane, probed with the indicated antibodies and detected by ECL (GE Healthcare Bio-Sciences Corp.). ECL signal and band density were quantified using Quantity One program (Bio-Rad). Protein expression was normalized to total protein or $\beta$-ACTIN.

\subsection{Statistical Analyses}

Data were analyzed using analysis of variance (one-way ANOVA) and multiplicities were adjusted by Tukey's method to control the family-wise error rate at 0.05 using JMP 9 software (SAS Institute, Inc; Cary, NC). $P \leq 0.05$ was defined as statistically significant in these studies. Hypothesis testing of synergistic effect were tested by interaction contrasts. Differences in survival between groups were compared by log-rank test. SAS 9.3 software (SAS Institute, Inc; Cary, NC) were used for data analysis.

\section{Results}

\subsection{M-CSF-R Driven Myr-Akt Induces an M2 Macrophage Tropism in Lung}

Since bone marrow-derived macrophages (BMM) collected from mice expressing Myr-Akt driven by the M-CSF-R promoter had increased survival [40], we first checked if Myr-Akt mice produced more BMM than WT mice. Penetrance 
of the Myr-Akt transgene correlated with transgene expression and splenomegaly. For this reason, classification as Myr-Akt genotype in this study was confirmed using both murine genotyping (Supplemental Figure 1(a)) and spleen size (Supplemental Figure 1(b)). Immunohistochemical staining for the macrophage marker $\mathrm{F} 4 / 80$ revealed a significant increase in macrophage numbers in the spleens of Myr-Akt mice compared to WT littermates ( $<$ < 0.001) (Figure 1(a), top and Figure 1(b)), however, we did not observe any differences in F4/80 expression in the lungs between these Myr-Akt and WT mice (Figure 1(a), bottom and Figure 1(b)). We next derived macrophages from bone marrow and measured F4/80 expression with flow cytometry. Our data suggest that Myr-Akt mice have increased numbers of F4/80+ BMMs compared to WT mice at day 1 $(\mathrm{p}=0.02)$ and day $2(\mathrm{p}=0.02)$ (Figure $1(\mathrm{c}))$. However, BMM numbers were similar after day 3 in culture (data not shown).

Since we did not observe more macrophages in the lungs of the Myr-Akt mice, we next asked if tissue macrophages from the lungs or spleens from Myr-Akt mice expressed a phenotype representing M2 phenotype [43] [44]. We isolated total RNA from whole lung or spleen tissue and performed qRT-PCR for the expression of M1 and M2 genes. There was no significant difference between Myr-Akt and WT mice in the M1 pro-inflammatory markers $I L-12$ and iNos (Figure 1(d), left), but there were significant increases in M2 anti-inflammatory genes $I L-10$ ( $p=0.047$ for lung and $\mathrm{p}=0.036$ for spleen) and Ym1 ( $p=0.01$ for lung and $p=0.05$ for spleen) of Myr-Akt compared to WT mice (Figure 1(d), right). Our data suggests that AKT activation driven by the M-CSF-R promoter in Myr-Akt mice drives an M2 tropism in both lung and splenic macrophages.

\section{2. $\alpha$-SMA+ and $\alpha$-SMA+/M-CSF-R+ Cell Influx in the Lungs of Unchallenged Myr-Akt Mice}

Since $\alpha$-SMA positive myofibroblasts mediate pulmonary fibrosis in human IPF [21] [45]. We next wanted to determine basal differences in $\alpha$-SMA expression in lung cells [46] from Myr-Akt and WT mice. Using immunostaining with the myofibroblast marker $\alpha$-SMA, we found more $\alpha$-SMA expression in lung tissue of Myr-Akt mice than WT mice ( $p=0.001)$ that were mostly confined in the epithelial and subepithelial tissues (Figure 2(a) and Figure 2(b)). This observation is supported by a significant increase in $\alpha$-Sma mRNA expression from whole lung tissue of Myr-Akt compared to WT mice $(p=0.05)$ (Figure 2(c)). Using flow cytometry, we also found increased numbers of cells expressing $\alpha$-SMA in the lungs of Myr-Akt mice compared to WT mice (mean $43.44 \% \pm 3.46 \%$ for Myr-Akt vs. $29.34 \% \pm 1.64 \%$ for WT; $p=0.02$ ) (Figure 2(d)). Since the Myr-Akt isoform is controlled by the M-CSF-R promoter, we next examined the population of $\alpha$-SMA+ myofibroblasts co-expressing M-CSF-R. We observed a significant increase in $\alpha$-SMA+/M-CSF- + + cells in the lungs of Myr-Akt mice compared to WT mice (mean $2.76 \% \pm 0.54 \%$ for Myr-Akt vs. $0.9 \% \pm 0.11 \%$ for WT; 

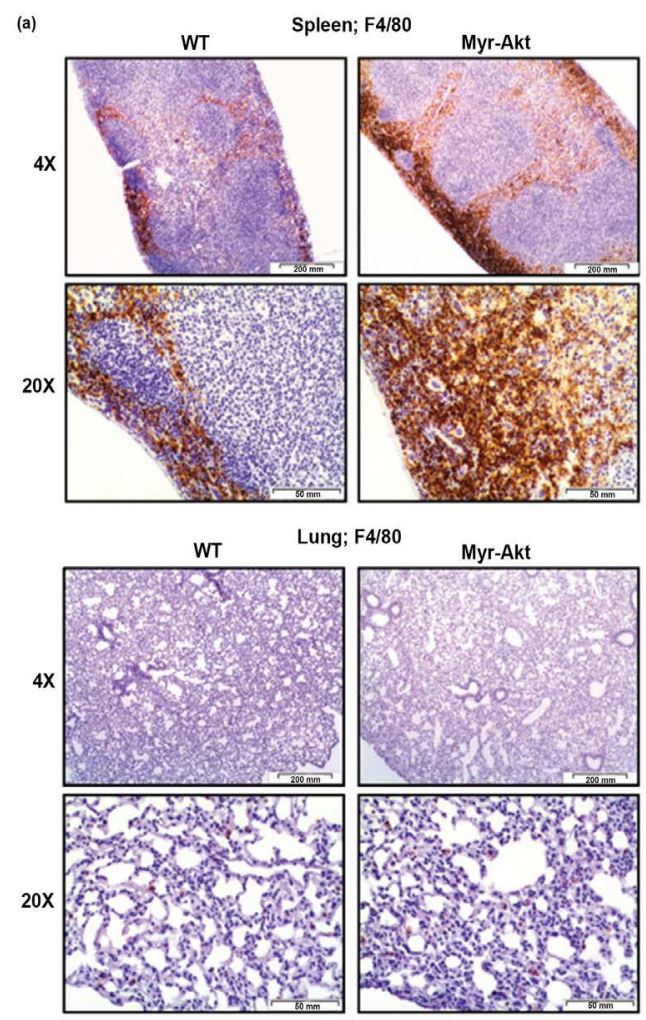

(d)

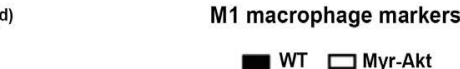

(b)

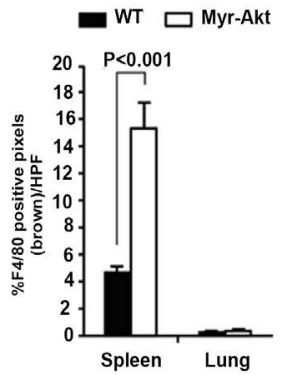

(c)

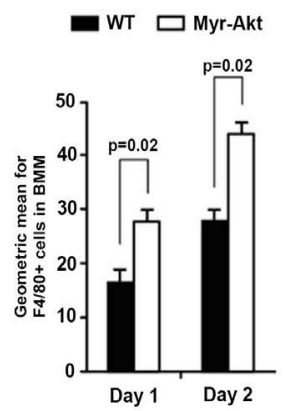

M2 macrophage markers

- WT $\square$ Myr-Akt
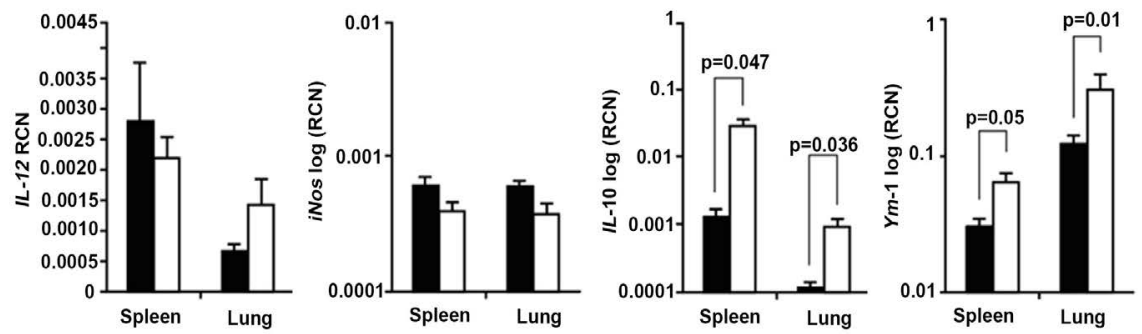

Figure 1. Myr-Akt mice demonstrate increased F4/80+ macrophages in the spleens and a M2 macrophage bias in the lungs. 6 - 8 week old Myr-Akt or WT mice were sacrificed and (a, top) spleens and (a, bottom) lungs were harvested, paraffin embedded, and subjected to F4/80 immunohistochemical staining for macrophages. Ten pictures per section were imaged using $4 \mathrm{X}$ and $20 \mathrm{X}$ objectives ( $40 \times$ and $200 \times$ magnification, respectively). (b) Images were quantified using histogram analysis feature in Adobe Photoshop CS5 software for percent brown stain (F4/80+ pixels) per high-power field (HPF) and expressed as mean \pm SEM. (c) BMMs were generated from Myr-Akt or WT littermate mice with recombinant murine $(\mathrm{rh}) \mathrm{CSF} 1(20 \mathrm{ng} / \mathrm{ml})$. Cells were removed using Accutase one and two days after seeding the progenitor cells (day 0). Cell surface antigen expression of F4/80 was assessed by flow cytometry. Data presented are the average geometric mean for F4/80 expression \pm SEM. (d) Total RNA was extracted from lung and spleen using TRIzol and cDNA synthesized from $1 \mu \mathrm{g}$ RNA for quantitative real-time (qRT)-PCR using primers specific for mouse IL12, iNos, IL10, or $Y m 1$. Data are expressed as relative copy number (RCN) (d, left) IL12 and iNos mRNA, or (d, right) IL10 mRNA and $Y m 1$ mRNA expression over the mean of endogenous control RNAs (Cap1, Rpl4 and Gapdh). Data represents the mean RCN \pm SEM. 5 pairs of age-matched littermate mice were used for each experiment. 

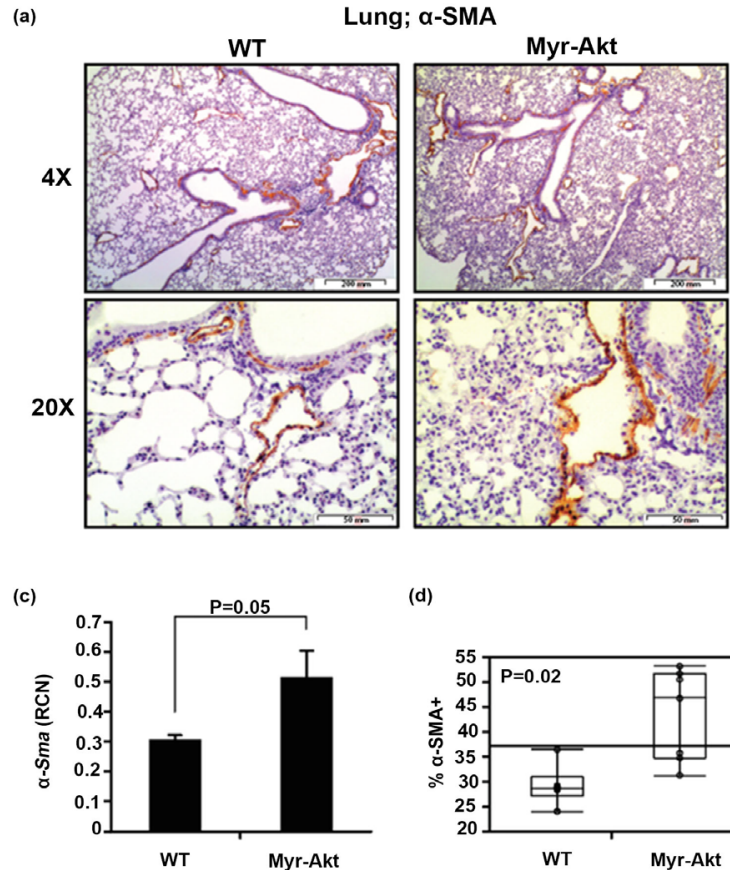

(d)

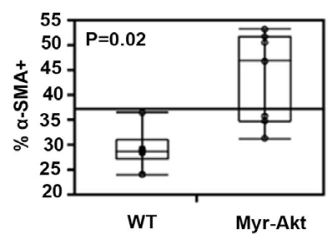

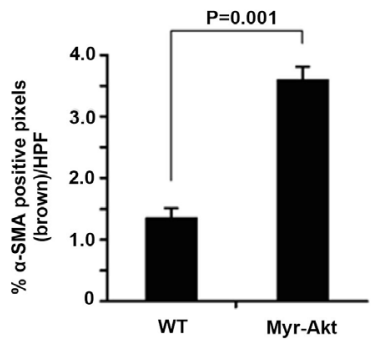

(e)

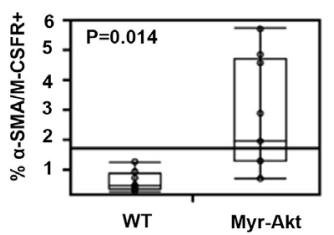

Figure 2. Differential myofibroblast expression in the lungs of Myr-Akt and WT mice. (a) 6 - 8 week old Myr-Akt or WT mice were sacrificed and lungs harvested, paraffin embedded, and subjected to $\alpha$-SMA immunohistochemical staining for myofibroblasts. Ten pictures per section were imaged using $4 \mathrm{X}$ and $20 \mathrm{X}$ objectives $(40 \times$ and $200 \times$ magnification, respectively). (b) Images were quantified by histogram analysis using Adobe Photoshop CS5 software and percent brown stain ( $\alpha$-SMA+ pixels) per high-power field (HPF) from at least five images per section are expressed as mean \pm SEM. (c) Total RNA was extracted from Myr-Akt or WT littermate mouse lungs and $\alpha$-SMA mRNA was measured by qRT-PCR. Data are expressed as relative copy number (RCN) of $\alpha$-SMA mRNA expression over the average of endogenous control RNAs (Cap1, Rpl4 and Gapdh). Data represent the mean RCN \pm SEM. Single-cell suspension from lung homogenate were immunostained using mouse $\alpha$-SMA antibodies or double-immunostained with $\alpha$-SMA and M-CSF-R (CD115) antibodies and analyzed by flow cytometry. (d) Data represents mean percent of $\alpha$-SMA+ cells and (e) $\alpha$-SMA+/M-CSF-R+ cells \pm SEM. 5 pairs of age-matched littermate mice were used for each experiment.

$\mathrm{p}=0.014$ (Figure 2(e)). These data suggest the Myr-Akt mice had increased basal concentrations of pulmonary myofibroblasts $(\alpha$-SMA+) co-expressing M-CSF-R than WT mice.

\subsection{Myr-Akt Mice have Altered Expression of Key Autophagy Genes in the Lung}

Autophagy is a process that maintains cell and tissue homeostasis by degrading and recycling cellular proteins and organelles during times of cellular stress. Autophagy activity is associated with organismal longevity and is reduced in lung tissue of IPF patients [29] [30] [31]. Because cellular autophagy is inhibited by the activation of the PI3K/AKT/mTOR pathway [47] [48] [49], we asked whether M-CSF-R-driven Myr-Akt impaired cellular autophagy in the lungs of the Myr-Akt mice. Following synthesis, the carboxy-terminal region of micro- 
tubule-associated protein light chain proLC3 is cleaved by the cysteine protease ATG4 generating the soluble cytosolic form of LC3A/B-I during autophagy, LC3A/B-I is converted to a membrane-bound LC3A/B-II form [50]. Since Cell Signaling Technology LC3B antibody mainly recognizes LC3BII, the hallmark of autophagy, we stained lung of Myr-Akt mice and their WT littermates with LC3B (Figure 3(a) and Figure 3(b)). Interestingly, we observed a significant decrease in LC3B in lungs from Myr-Akt mice compared to lungs from WT mice ( $\mathrm{p}=0.016)$, implying autophagy is impaired in the lung of Myr-Akt mice. We next analyzed key autophagy-regulating genes: Beclin-1 [29] and Lc3a and $L c 3 b$ [50] [51] by qRT-PCR. Beclin-1, Lc3a and $L c 3 b$ gene expression were significantly reduced in lungs from Myr-Akt mice compared to lungs from WT mice ( $\mathrm{p}=0.0025$ for Beclin-1, and $\mathrm{p}<0.001$ for Lc3a and $L c 3 b$ ) (Figure 3(c)). Similarly, BECLIN-1 and LC3A/B-II protein was also significantly reduced in the lungs of Myr-Akt mice compared to lungs from WT mice ( $p<0.01$ for BECLIN-1, $\mathrm{p}=0.09$ for LC3A/B-I and $\mathrm{p}=0.014$ for LC3A/B-II) (Figure 3(d)). Because inhibition of autophagy increases autophagy receptor p62 protein accumulation [52], we also assessed the expression of polyubiquitin-binding protein $\mathrm{p} 62 / \mathrm{SQSTM} 1$ and observed an increased accumulation of $\mathrm{p} 62$ protein in the lungs of Myr-Akt mice (Figure 3(d), p = 0.07). Even though p62 protein serves as an autophagy marker, it can be regulated at both transcriptional and post-translational levels [53], therefore we measured p62 mRNA by qRT-PCR from Myr-Akt and WT mice lungs and found no change on p62 mRNA, indicating that p62 is regulated at a post-translational level and the accumulation of p62 protein is due to low autophagy (Figure $3(\mathrm{e})$ ). Further, since AKT positively regulates mTOR activity, we confirmed an elevation of phosphor-mTOR in the lungs of Myr-Akt mice (Figure 3(f), p = 0.029). Taken together, these data suggest that the lungs of Myr-Akt mice have reduced basal autophagy and increased mTOR activity compared to lungs of WT mice.

\subsection{Myr-Akt Mice Produce Increased Numbers of Circulating Fibrocytes in Vivo}

Bone-marrow derived circulating fibrocytes can traffic to the lung during the pathogenesis of pulmonary fibrosis [24] [28]. We inquired whether Myr-Akt mice could generate more circulating fibrocytes, in vivo. We generated fibrocytes from mouse PBMCs cultured in fibrocyte-deriving medium and observed more spindle-shaped fibroblast-like cell morphology from Myr-Akt mice compared with WT mice (Figure 4(a)). Since CD45+/COL1+/CXCR4+ fibrocytes were expanded in the bone marrow and have been reported to contribute to bleomycin-induced pulmonary fibrosis [24], we next stained the PBMC-differentiated cells with antibodies specific for CD45, Collagen I, and CXCR4 (Figure 4(b)). Our data suggest Myr-Akt mice generate significantly more circulating CD45+/COL1+/CXCR4+ fibrocytes than WT mice $(\mathrm{p}=0.05)$, basally. 


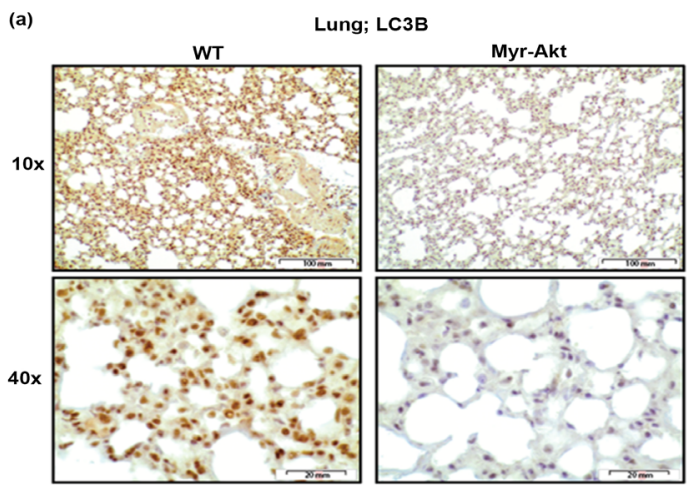

(d)
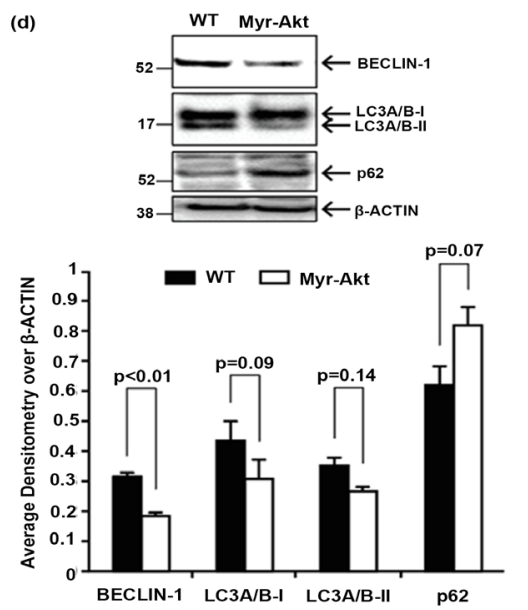

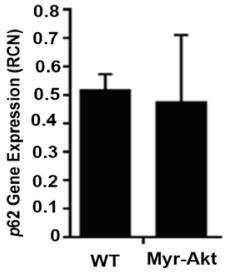

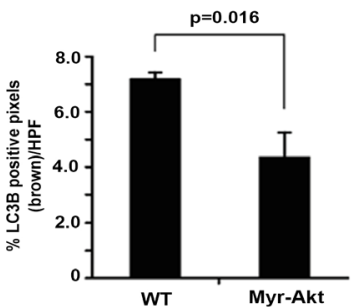

(c) WT $\square$ Myr-Akt

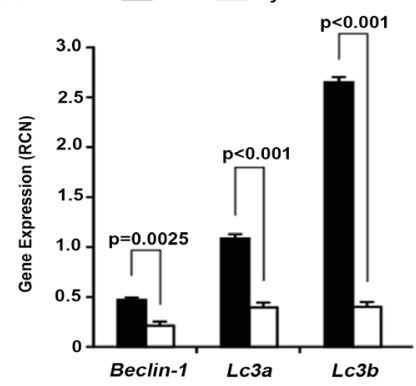

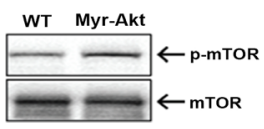

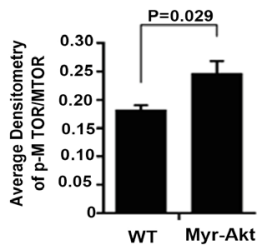

Figure 3. Reduced autophagy in the lungs of Myr-Akt mice. (a) 6 - 8 week old Myr-Akt or WT mice were sacrificed and lungs harvested, paraffin embedded, and subjected to LC3B immunohistochemical staining. Ten pictures per section were imaged using 10X and 40X objectives (100x and 400× magnification, respectively). (b) Images were quantified using histogram analysis feature in Adobe Photoshop CS5 software for percent brown stain (F4/80+ pixels) per high-power field (HPF) from at least five images per section and expressed as mean \pm SEM. (c) Total RNA was extracted from homogenized lungs using TRIzol. cDNA was synthesized and qRT-PCR performed using primers specific for mouse Beclin-1, Lc3a, and $L c 3 b$ mRNAs. Data are expressed as relative copy number (RCN) of mRNA expression over average endogenous control RNAs (Cap1, Rpl4 and Gapdh). Data represents the mean RCN \pm SEM. (d) Total protein were isolated and equal amounts of protein separated on a $4 \%-12 \%$ SDS-PAGE gel and immunoblotted for BECLIN-1, LC3A/B-I and LC3A/B-II, and $\beta$-ACTIN. (e) Total RNA was extracted from homogenized lungs using TRIzol. cDNA was synthesized and qRT-PCR performed using primers specific for mouse $p 62 / S Q S T M 1$ mRNA. Data are expressed as relative copy number (RCN) of mRNA expression over average endogenous control RNAs (Cap1, Rpl4 and Gapdh). Data represents the mean RCN \pm SEM. (f) Immunoblot for phosphor-mTOR (ser2448) and mTOR (Cell Signaling Technology). Bands were quantified using densitometry by comparing band density over $\beta$-ACTIN or total mTOR in each respective lane. Data are expressed as the mean densitometry value \pm SEM. 4 pairs of age-matched littermate mice were used for western analysis and 5 pairs of age-matched littermate mice were used for gene expression. 
(a)

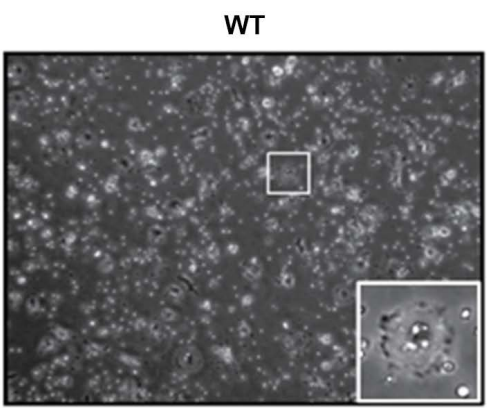

Myr-Akt

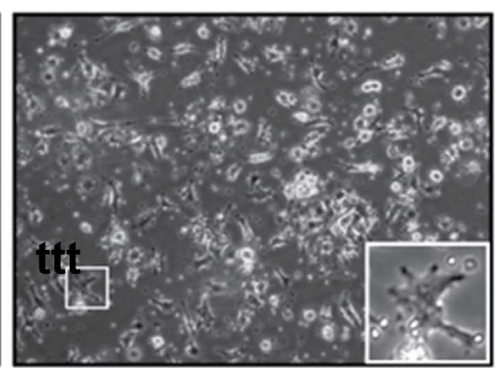

(b)

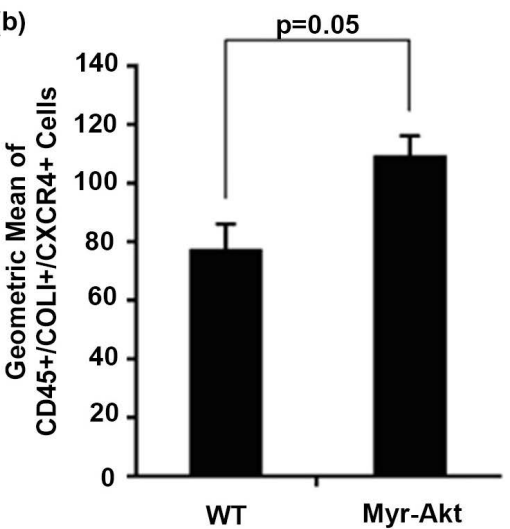

Figure 4. Myr-Akt mice have elevated circulating fibrocytes compared to WT mice. (a) 6 - 8 week old Myr-Akt or WT mice were sacrificed and blood was collected from cardiac puncture. PBMCs were isolated and cultured in FibroLife media for 5 days to generate mouse fibrocytes. Images were taken at day 5 using a 10X objective (100x magnification). (b) Cells were removed using Accutase and equal number of cells were stained with CD45 and CXCR4, then permeabilized/fixed and stained for Collagen I. The cells were analyzed by flow cytometry and fibrocytes were identified as CD45+/COLI+/CXCR4+ populations. Data represents mean percent of fibrocytes \pm SEM from 5 mice per group.

\subsection{Bleomycin Challenge Enhances Collagen Deposition in the Lungs and Reduces Survival in Myr-Akt Mice}

Because Myr-Akt macrophages express an M2 tropism, increased $\alpha$-SMA+ myofibroblasts, increased circulating CD45+/COL1+/CXCR4+ fibrocytes, and reduced autophagy-related gene expression, we hypothesized that Myr-Akt mice would develop more fibrosis in response to repeated administration of bleomycin.

Kaplan-Meier survival analysis (Figure 5(a)) illustrates decreased survival in Myr-Akt mice challenged with bleomycin compared to bleomycin-treated WT mice $(p=0.0284)$. While there was no mortality in the Myr-Akt or WT mice groups treated with PBS (vehicle), the Myr-Akt mice started to die at day 7 post-bleomycin treatment while all WT mice survived until day 28 on the bleomycin protocol. The mice receiving bleomycin and surviving to day 33 showed typical fibrosis in the lung compared to vehicle-treated mice, including blue stained pixels (Trichrome) as an indication of collagen deposition (Figure 5(b) and Figure 5(c)) and significant inflammation (Figure 5(d)). Bleomycin 

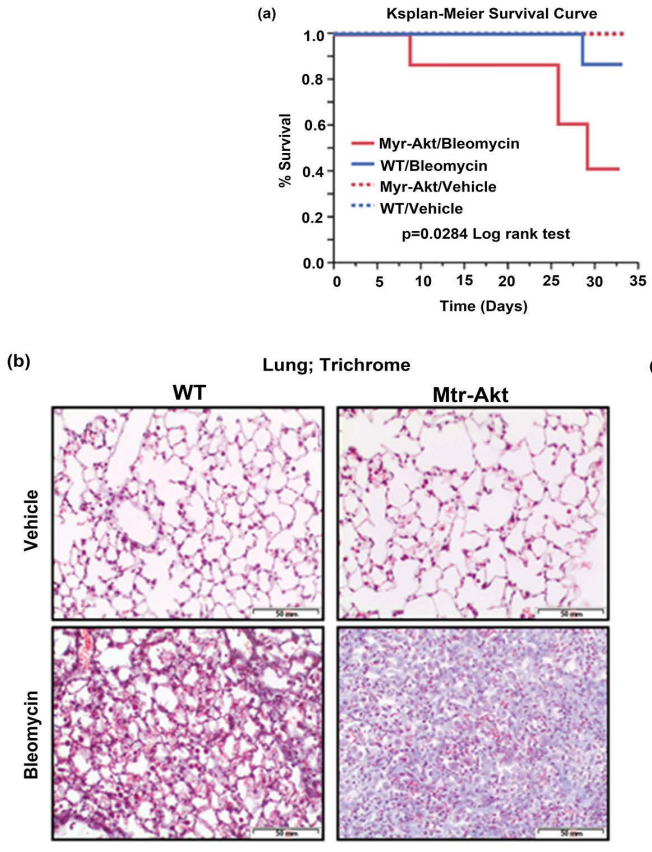

(d)
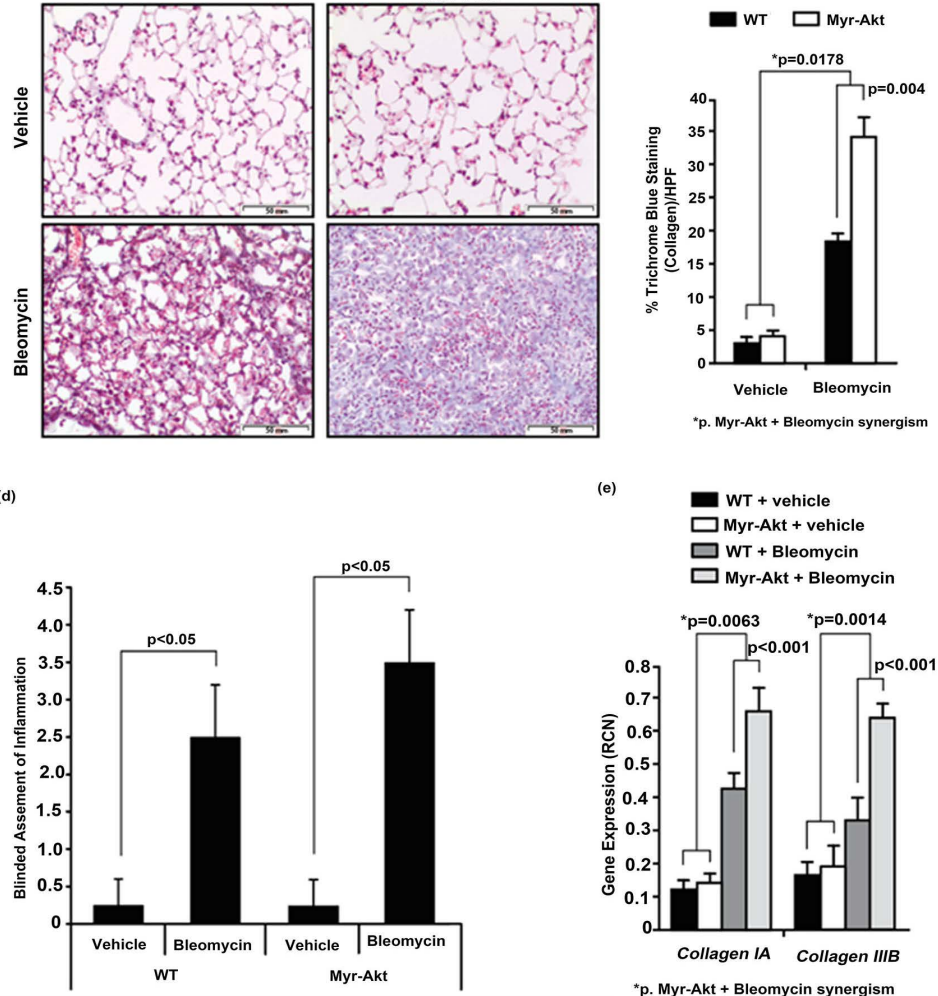

Figure 5.Bleomycin induces collagen deposition in the lungs and reduces survival in Myr-Akt mice. Myr-Akt and wild type (WT) littermate mice were treated twice a week with bleomycin $(0.035 \mathrm{U} / \mathrm{g})$ or PBS (vehicle) for 4 weeks, as described in Material and Methods. (a) Mouse survival was recorded and plotted using a Kaplan-Meier curve. Myr-Akt mice had worse survival than WT mice in response to bleomycin treatment. $\mathrm{N}=$ 7 mice for each WT and Myr-Akt mouse group. (b) All surviving mice were sacrificed at day 33 and the lungs formalin-fixed, sectioned, and stained with Masson's Trichrome. Five images per slide were captured. Red staining represents keratin, light red and pink staining represents cytoplasm, dark brown represents cell nuclei, and blue staining represents collagen. The images are representative images from 3 mice per group using a $20 \mathrm{X}$ objective (200x magnification). (c) The images were quantified blindly by histogram analysis using Adobe Photoshop CS and the percent of blue staining per high-power field (HPF) from ten images and expressed as mean \pm SEM. (d) Lungs from WT and Myr-Akt mice subjected to PBS or bleomycin stained with H\&E, Trichrome, and F4/80 were evaluated by a board-certified pathologist for inflammation score ( 0 to 5 point scale). (e) Total RNA was extracted from homogenized lung tissue using TRIzol. cDNA was synthesized and qRT-PCR performed for mouse collagen IA and IIIB. Data are expressed as mean relative copy number (RCN) of mRNA expression over average endogenous control RNAs (Capl, Rpl4 and Gapdh). Data represents the mean RCN \pm SEM and N $=4$ mice per group. 
increased collagen deposition in the lungs of WT mice by $15 \%$ (bleomycin vs. vehicle in WT, $\mathrm{p}<0.001$ ), while Myr-Akt increased the bleomycin effect to $28 \%$ (bleomycin vs. vehicle in Myr-Akt, $\mathrm{p}=0.004$ ), and a statistical synergistic test showed that this enhancement was significant $(p=0.0178)$. Moreover, bleomycin treatment up-regulated collagen IA and IIIB mRNAs by 4 -fold and 3-fold in WT (both $p<0.001$, compared with vehicle), while Myr-Akt increased the bleomycin effect to 6-fold and 5-fold, respectively (bleomycin vs. vehicle in Myr-Akt, both $p<0.0001)$. A statistical synergistic test showed that this enhancement was significant $(\mathrm{p}=0.0063$ for collagen IA, $\mathrm{p}=0.0014$ for collagen IIIB) (Figure 5(e)). Our results suggest that Myr-Akt enhances the bleomycin effect leading to the increased lung fibrosis and decreased overall survival.

\subsection{Myr-Akt mice Demonstrate Increased CD45 and F4/80+ Cells after Bleomycin Challenge}

To determine if Myr-Akt mice had enhanced lung inflammation, we immunostained mouse lungs using the leukocyte marker CD45 [54] and tissue macrophage marker F4/80 [16]. More CD45+ cells are present in Myr-Akt mice compared with WT $(\mathrm{p}=0.05)$ (Figure 6(a) and Figure 6(b)). As expected, bleomycin treatment significantly increased CD45+ cells in the lungs of WT and Myr-Akt mice (both $\mathrm{p}<0.01$ ). Additionally, more CD45+ cells were present in the lungs of Myr-Akt mice compared to the lungs of WT mice after bleomycin challenge ( $p=0.001$ ) (Figure 6(a) and Figure 6(b)). Importantly, unlike CD45+ cells, and as seen in Figure 1(a) and Figure 1(b), the F4/80+ cells are comparable between Myr-Akt and WT mice (Figure 6(c) and Figure 6(d)). After bleomycin challenge, more F4/80+ cells were present in the lungs of Myr-Akt mice than WT mice $(\mathrm{p}=0.01)$. As expected, bleomycin increased $\mathrm{F} 4 / 80+$ cells in the lungs of WT mice by 6 -fold $(p<0.001)$, while Myr-Akt increased the bleomycin effect by 8 -fold (bleomycin vs. vehicle in Myr-Akt). Although not quite statistically different, Myr-Akt and bleomycin exerted a marked synergism on F4/80 expression $(p=0.0708)$ (Figure $6(\mathrm{~d})$ ). These data suggest that bleomycin-induced lung fibrosis may involve recruited hematopoietic cells, specifically M2 macrophages.

\subsection{Bleomycin Suppresses Autophagy Gene Expression in the Lungs}

Reduced levels of autophagy in IPF induce epithelial cell senescence and accelerate lung fibroblast differentiation into myofibroblasts [29]. Our data suggest that Myr-Akt mice both basally and post-bleomycin contained more $\alpha$-SMA+ lung myofibroblasts and fewer autophagy-related genes expressed in lung tissue than WT mice. Specifically, we initially observed (Figure 3) that basal Myr-Akt expression decreased the key autophagy genes Beclin-1, LC3a and LC3b compared to WT. This data was confirmed as vehicle-treated Myr-Akt mice had significantly reduced Beclin-1, $L C 3 a$ and $L C 3 b$ mRNAs in whole lung tissue compared to WT mice ( $p<0.001$ for Beclin-1, $L C 3 a$, and $L C 3 b$ ). Predictably, bleomycin 


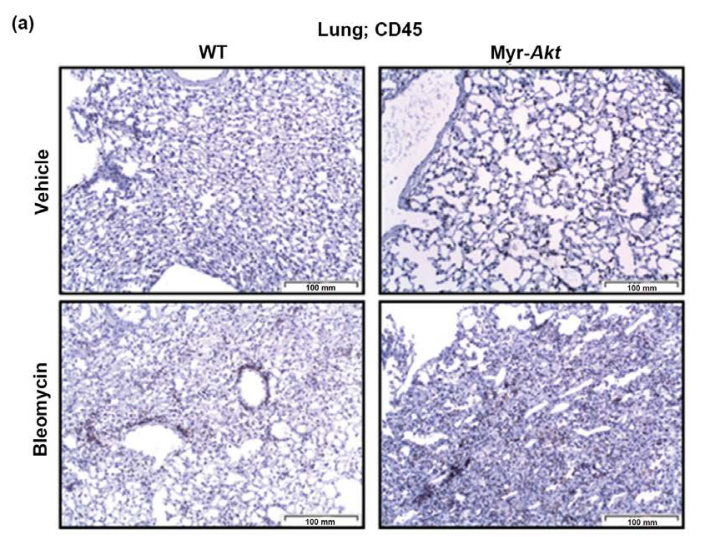

(c)

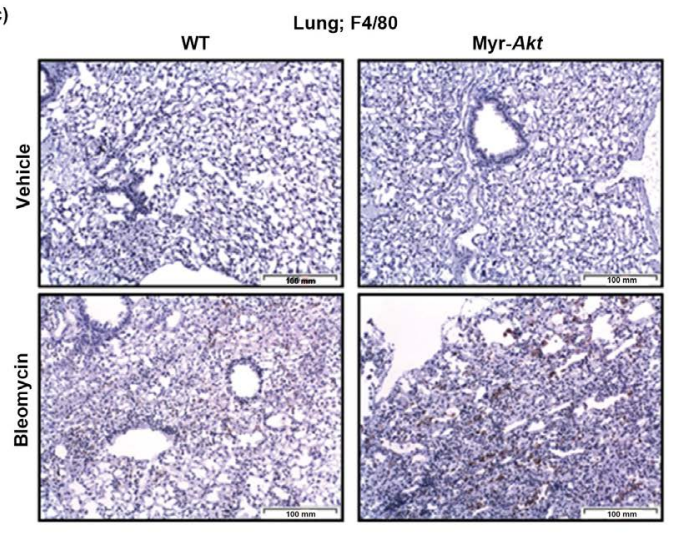

(b)

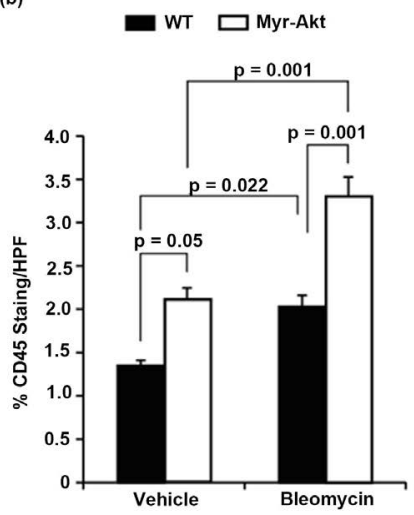

(d)

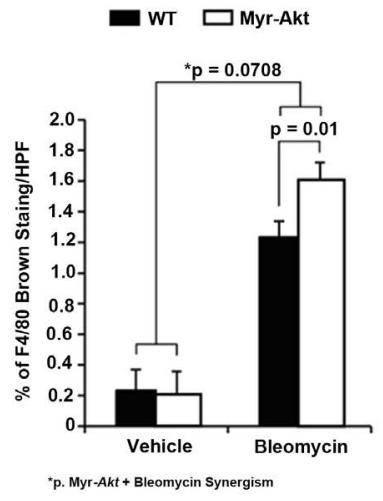

Figure 6. Bleomycin increases CD45+ and F4/80+ cells in the lungs of Myr-Akt mice. Mice surviving the bleomycin or PBS protocol were sacrificed at 33 days and lungs harvested and subjected to immunohistochemical staining. Ten pictures per slide were imaged. Brown pixels represent (a) CD45+ or (c) F4/80+ cells. Images are representative of three mice per group. The images were quantified blindly by histogram analysis using Adobe Photoshop CS and the percent brown stain per high-power field (HPF) from at least five images per section using a $10 \mathrm{X}$ objective $(100 \times$ magnification $)$ and are expressed as the mean \pm SEM (b) and (d).

treatment in both WT and Myr-Akt mice further reduced Beclin-1 mRNA ( $p<$ 0.001 for vehicle vs. bleomycin in WT mice; $p<0.006$ for vehicle vs. bleomycin in Myr-Akt mice); LC3a mRNA ( $p<0.001$ for vehicle vs. bleomycin in WT mice; $p<0.001$ for vehicle vs. bleomycin in Myr-Akt mice); and $L C 3 b$ mRNA ( $p$ $<0.001$ for vehicle vs. bleomycin in WT mice; $p<0.001$ for vehicle vs. bleomycin in Myr-Akt mice) compared to sample-matched vehicle control (Figure 7). Interestingly, a synergistic decrease of autophagy genes was observed after bleomycin treatment in Myr-Akt mice ( $\mathrm{p}<0.001$ for beclin-1, LC3a and LC3b) (Figure 7).

\section{Discussion}

We previously reported that CSF1 plays an important role in pulmonary fibrosis in both mice and IPF patients [38]. However, that report lacked critical mechanistic data about how CSF1 and M-CSF-R-mediated signaling contributes to 


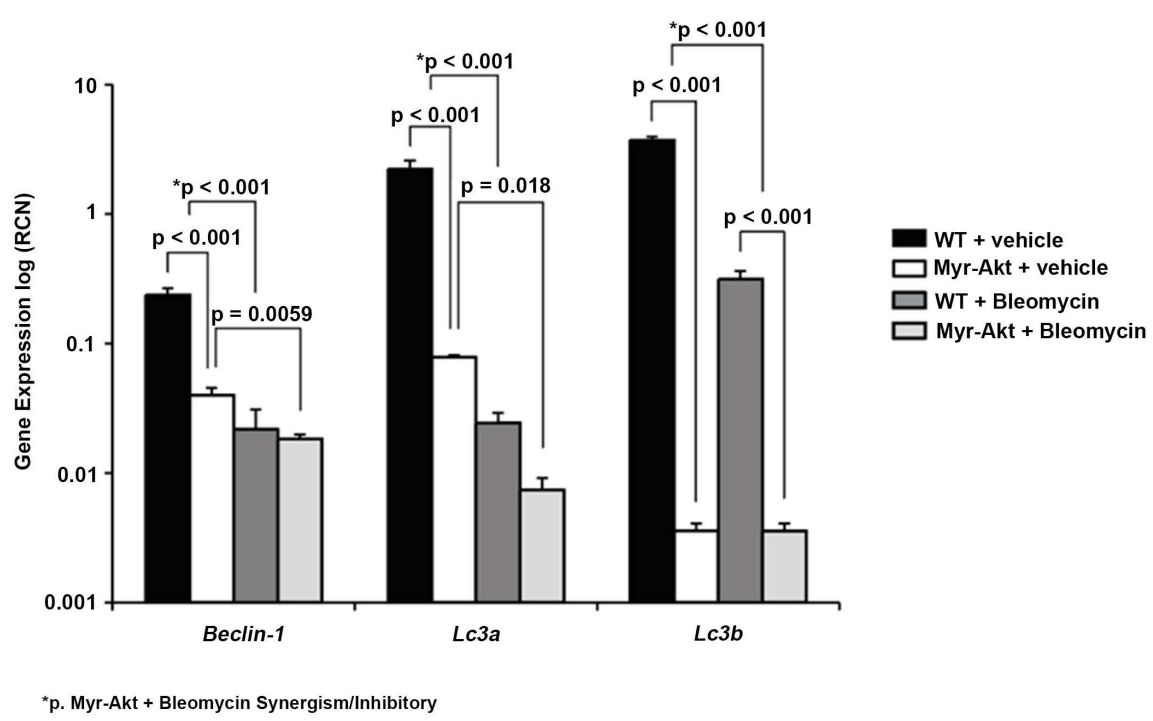

Figure 7. Bleomycin suppresses autophagy gene expression in the lungs of Myr-Akt mice. Total RNA was extracted from paraffin embedded lung tissue section with RecoverAll Total Nucleic Acid Isolation Kit for FFPE according to manufacturer's protocol. cDNA was synthesized and qRT-PCR performed for mouse Beclin-1, Lc3a, and $L c 3 b$. Data are expressed as relative copy number (RCN) of mRNA expression over average of endogenous control RNAs (Capl, Rpl4 and Gapdh.). Data represents the mean RCN \pm SEM and $\mathrm{N}=4$ mice per group.

lung fibrosis. To accomplish an understanding of how M-CSF-R and AKT activation may contribute to fibrosis, we used a novel transgenic mouse model which expresses a myristoylated and constitutively-active AKT isoform under the control of the M-CSF-R promoter to investigate the connections between cellular CSF1 signaling and the development of pulmonary fibrosis. Interestingly, we did not observe basal structural differences in the lungs of the transgenic or wild type mice as shown by Hematoxylin \& Eosin (H\&E) staining (Supplemental Figure 2(a)). However, compared to lung tissue from WT mice, Myr-Akt mice lungs contained macrophages that expressed a basal M2 tropism, exhibited a higher number of lung cells co-expressing M-CSF-R and $\alpha$-SMA, contained increased numbers of circulating fibrocytes, and had reduced key autophagy gene expression: $L c 3 a / b$, Beclin-1 and p62 at both the mRNA and protein levels. Further, when challenged with bleomycin, Myr-Akt mice had more lung fibrosis, inflammation, myofibroblasts, less autophagy and shorter life span than WT mice given bleomycin. Interestingly, we observed a synergistic effect of Myr-Akt expression and bleomycin challenge on cellular and molecular pathways that regulate lung pathology and fibrosis. Our study provides insight into understanding a critical role for CSF1-induced AKT activation in the predisposition to lung fibrosis.

While we are excited about the potential of M-CSF-R and AKT targeting in lung fibrosis, we acknowledge limitations in the Myr-Akt mouse as a model for human lung fibrosis. First, the penetrance of Myr-Akt expression is difficult to 
ascertain in these mice solely by copy number. Thus, to select mice that had maximum penetrance of the Myr-Akt construct, we first genotyped the mice and then classified those Myr-Akt mice with enlarged spleens as Myr-Akt mice displaying the constitutively-active AKT phenotype as splenomegaly is a characteristic of enhanced macrophage accumulation in these mice when compared to wild type mice. We further stained spleen with the macrophage marker F4/80 and confirmed Myr-Akt mice had significantly higher level of F4/80 accumulation in the spleen than WT mice. Finally, we assayed lungs, spleens, and bone marrow from both groups of mice and found augmented phosphorylation of AKT and ERK in the Myr-Akt mice (Supplemental Figure 3). Due to technical limitations, we could not effectively obtain enough M-CSF-R+ cells from mouse lungs. We would expect to observe greater differences at the gene and protein levels between Myr-Akt mice and WT mice with M-CSF-R+ cells.

This unique model provides insight into the role of M-CSF-R in lung fibrosis. Despite the limitations of mouse models to emulate human lung fibrosis, we gained valuable insight from this study. First, M-CSF-R-induced AKT activation enhanced basal lung M2 macrophage tropism in Myr-Akt mice suggesting that activation of M-CSF-R- and AKT-driven genes may predispose a host to a fibrotic response after secondary insult or injury. In human fibrotic organ tissue, M2 macrophages contribute to the regulation of inflammation, tumor growth, stromal formation, and clearance of cell debris in the ECM [16].

In addition to our observations about the role of M-CSF-R activation in IPF [38], another study demonstrated elevated M2 genes CCL18, CCL22, and CD206 from alveolar macrophages of IPF patients. In this study, the authors proposed $L-4$ and $I L-10$ are responsible for the M2 phenotype shift [55]. Most importantly, our findings suggest a link between M-CSF-R-regulated AKT activation and an M2 macrophage polarity as whole lung tissue from Myr-Akt mice had increased IL-10 mRNA and an M2 macrophage polarization in both lungs and spleens. Admittedly, alveolar macrophages are not the only producers of IL-10, and increases in IL-10 mRNA from whole lung tissue could be from other inflammatory cells as well as type I alveolar epithelial cells in this model. Studies are underway to confirm the sources of lung IL-10 in the mouse model. Indeed, the negative regulator of PI3K/AKT, src homology 2-containing inositol phosphatase (SHIP), interferes with lung M2 macrophage skewing as peritoneal and alveolar macrophages from SHIP1-/- mice express a strong M2 gene signature in terms of arginase-1 and $Y m-1$ mRNAs along with M2-mediated lung pathology [44]. Bone marrow-derived macrophages from SHIP1-/- mice display an M2 phenotype only upon TGF- $\beta$ or IL-3 stimulation [14] [43]. Furthermore, the PI3K inhibitor LY294002 decreases the expression of M2 genes from alveolar macrophages [2] [14]. It is interesting that we did not observe any significant morphological changes or increase in macrophage numbers in the lungs from the Myr-Akt compared to WT mice (Figures 1(a), bottom and Figures 1(b)). Therefore, our data of macrophage M2 phenotype tropism, increased myofibroblast and circulating fibrocytes population, and reduced autophagy genes 
suggest a potential priming effect driven by M-CSF-R and AKT activation that favors a lung environment that is primed for fibrosis.

The current paradigm in pulmonary fibrosis suggests that in a predisposed host, repeated subclinical lung injury and epithelial damage results in recruitment and activation of highly contractile and fibrotic myofibroblasts [2] [56]. Interestingly, phosphatase and tensin homolog (PTEN), a negative regulator of AKT activity, is down-regulated in myofibroblasts [56] isolated from IPF patients [9]. We observed elevated numbers of basal $\alpha$-SMA+ myofibroblasts in untreated Myr-Akt lungs. Moreover, myofibroblasts have been shown to be more resistant to apoptosis than normal fibroblasts, which may interrupt epithelial cell repair [19] [30] [57]. As constitutively-active AKT was observed in spleens, lungs, and bone marrow of Myr-Akt mice (Supplemental Figure 3), we speculated that persistent AKT activation may lead to myofibroblast survival, differentiation, and ultimately organ fibrosis. Of note, we observed increases in $\alpha$-SMA/M-CSF-R double-positive cells in the lung of Myr-Akt mice. This observation suggests that as part of the priming effect, either proliferating smooth muscle cells known to express M-CSF-R [22] differentiate into myofibroblasts, or macrophages possibly trans-differentiate into myofibroblasts. These questions require further investigation to clarify the hematopoietic or mesenchymal origin of these cells.

Increasing evidence indicates detection of fibrocytes in fibrotic lungs. Fibrocytes are important sources of fibroblasts and myofibroblasts during tissue repair and remodeling processes [24]. Indeed, a key marker for fibrocytes, CXCR4 expression, is regulated by the PI3K/AKT/mTOR pathway and hypoxia as inhibiting PI3K/AKT/mTOR activity reduced PDGF and hypoxia-induced CXCR4 [27]. These data support increases in circulating fibrocytes in our Myr-Akt mice. Interestingly, a recent report suggests that fibrocytes do not contribute to significant type I collagen deposition in fibrotic lungs and that they may play an indirect role in fibrosis by orchestrating recruitment of other cells [58]. Studies to investigate the role of $\mathrm{AKT}$ in the differentiation of macrophages into fibroblasts and their paracrine effects on fibroblasts are ongoing.

In addition to the predisposed M2 polarity, and myofibroblast and fibrocyte presence, we also found cells from lungs of Myr-Akt mice had altered expression of key autophagy-regulatory genes, indicating reduced autophagy. Since autophagy is a critical pathway for cellular longevity and homeostasis, and is reduced in lungs of IPF patients, understanding the molecular regulation of this pathway may contribute to effective pharmacological intervention. Based on existing literature, we chose to measure LC3-II (lipidated/autophagosome membrane associated form of LC3), Beclin-1 and p62 (ubiquitin binding autophagy substrate receptor) proteins as markers for autophagic. Use of three markers to assess autophagic helps overcome the limitations of any one single marker in assessing such a complex system, facilitating more accurate interpretation of results. Our results indicate that lung tissue from Myr-Akt mice had reduced autophagy. Consistent with our observation in Myr-Akt mice, others demonstrate that inhi- 
biting autophagy by knocking down $L c 3 b$ and Atg 5 mRNAs enhance expression of $\alpha$-SMA and type I collagen in lung fibroblasts [29]. Similarly, inducing autophagy by inhibiting mTOR activity in lung fibroblasts with rapamycin reduces cellular $\alpha$-SMA and fibronectin expression [30] linking autophagy and $\alpha$-SMA expression with lung fibrosis in Myr-Akt mice. Of note, in addition to qRT-PCR for expression of lung collagen mRNAs, we elected to use Trichrome staining in lieu of Sircol or hydroxyproline assay on whole lung tissue to determine potential difference in location of collagen deposition. Further, we failed to assess autophagic flux with lysosome inhibitors, in vivo. Autophagic flux is the complete process of autophagy from phagophore formation to substrate degradation and release of breakdown products. Measuring autophagy flux will be included in our future autophagy analysis.

Interestingly, the regulation of autophagy may be cell-specific. Araya et al. reported that activating autophagy in human bronchial epithelial cells suppresses endoplasmic reticulum (ER) stress and cell senescence [29]. In contrast, inhibiting autophagy also promotes myofibroblast differentiation in the lungs of IPF patients [29]. Our study showed a reduction in key autophagy genes, Beclin-1, $L c 3 a$ and $L c 3 b$ in Myr-Akt mouse lungs. Several lines of evidence indicate that autophagy, apoptosis, and senescence are tightly regulated to maintain cellular and organ homeostasis. For example, reduced apoptosis in lung myofibroblasts from human IPF samples results in dysregulated lung repair [59] correlating with the inhibition of autophagy in IPF lung tissue [30] [31] [47]. Importantly, AKT-induced mTOR activation is critical in reducing autophagy as rapamycin has been shown to induce autophagy [47]. We observed elevated mTOR activity in lungs of Myr-Akt mice suggesting decreased autophagy via the AKT/mTOR pathway. Furthermore, in our Myr-Akt murine model, constitutive AKT activation correlated with reduced autophagy gene expression, which was further reduced by bleomycin challenge. It is plausible that the combination of constitutively-active AKT and bleomycin creates a scenario where clearance of unwanted proteins and organelles is hindered, leading to reduced survival times in Myr-Akt mice. We are currently investigating if AKT activation in macrophages, and the observed changes in lung expression of autophagy markers, are directly attributable to changes in the macrophages themselves or if this AKT activity translates into alterations of other cell phenotypes.

This report provides links between M-CSF-R-induced AKT activity and M2 macrophage bias, myofibroblast marker expression, and decreased autophagy gene expression. We used a transgenic murine model to demonstrate a pro-fibrotic role for CSF1 and AKT in the lungs, and believe that these observations may be applicable to clinical lung disease, as we showed overexpressing AKT predisposes lungs to fibrosis in the presence of bleomycin. This prediction is based on our previous data that showed CSF1 is important in IPF, and we surmise that this report clarifies a potential mechanism for this effect as a priming induced by CSF1 to predispose lung fibrosis in humans. 


\section{Acknowledgements}

The authors appreciate the assistance provided by Leeanne Garrett and Christie Newland of the Animal Core Facility at DHLRI who maintained the mouse colonies and helped perform mouse surgeries. The authors also acknowledge the Immunohistochemistry Core Facility at The Ohio State University Veterinarian School for assistance with tissue sectioning and staining. Melissa G. Piper passed away in December 2013. This work was supported by research funding from The National Institutes of Health/National Heart, Lung, and Blood Institute (NHLBI) HL067176, HL109481 and HL102464 (C.B.M.).

\section{Author Contributions}

Conception and design: C.B.M, M.G.P., T.D.E., Y.W.; acquisition of data: Y.W., D.D., T.B., E.E.; analysis and interpretation of data: C.B.M., T.D.E., Y.W., D.D., X.M.; writing and editing of the manuscript: D.D., Y.W., T.D.E., C.B.M.

\section{Conflicts of Interest}

The authors report no conflicts of interest in this study.

\section{References}

[1] Raghu, G., Weycker, D., Edelsberg, J., Bradford, W.Z. and Oster, G. (2006) Incidence and Prevalence of Idiopathic Pulmonary Fibrosis. American Journal of Respiratory and Critical Care Medicine, 174, 810-816. https://doi.org/10.1164/rccm.200602-163OC

[2] Coward, W.R., Saini, G. and Jenkins, G. (2010) The Pathogenesis of Idiopathic Pulmonary Fibrosis. Therapeutic Advances in Respiratory Disease, 4, 367-388. https://doi.org/10.1177/1753465810379801

[3] Franke, T.F., Kaplan, D.R. and Cantley, L.C. (1997) PI3K: Downstream AKTion Blocks Apoptosis. Cell, 88, 435-437. https://doi.org/10.1016/S0092-8674(00)81883-8

[4] Hunter, M., Wang, Y., Eubank, T., Baran, C., Nana-Sinkam, P. and Marsh, C. (2009) Survival of Monocytes and Macrophages and Their Role in Health and Disease. Frontiers in Bioscience, 14, 4079-4102. https://doi.org/10.2741/3514

[5] Lu, Y., Azad, N., Wang, L., Iyer, A.K., Castranova, V., Jiang, B.H. and Rojanasakul, Y. (2010) Phosphatidylinositol-3-Kinase/Akt Regulates Bleomycin-Induced Fibroblast Proliferation and Collagen Production. American Journal of Respiratory Cell and Molecular Biology, 42, 432-441. https://doi.org/10.1165/rcmb.2009-0002OC

[6] Huang, J.F., Chuang, Y.H., Dai, C.Y., Yu, M.L., Huang, C.F., Hsiao, P.J., Hsieh, M.Y., Huang, C.I., Yeh, M.L., Yang, J.F., Lin, Z.Y., Chen, S.C. and Chuang, W.L. (2011) Hepatic Akt Expression Correlates with Advanced Fibrosis in Patients with Chronic Hepatitis C Infection. Hepatology Research, 41, 430-436. https://doi.org/10.1111/j.1872-034X.2011.00786.x

[7] Le Cras, T.D., Korfhagen, T.R., Davidson, C., Schmidt, S., Fenchel, M., Ikegami, M., Whitsett, J.A. and Hardie, W.D. (2010) Inhibition of PI3K by PX-866 Prevents Transforming Growth Factor-Alpha-Induced Pulmonary Fibrosis. The American Journal of Pathology, 176, 679-686. https://doi.org/10.2353/ajpath.2010.090123

[8] Vittal, R., Horowitz, J.C., Moore, B.B., Zhang, H., Martinez, F.J., Toews, G.B., Standiford, T.J. and Thannickal, V.J. (2005) Modulation of Prosurvival Signaling in Fi- 
broblasts by a Protein Kinase Inhibitor Protects against Fibrotic Tissue Injury. The American Journal of Pathology, 166, 367-375.

https://doi.org/10.1016/S0002-9440(10)62260-2

[9] Xia, H., Khalil, W., Kahm, J., Jessurun, J., Kleidon, J. and Henke, C.A. (2010) Pathologic Caveolin-1 Regulation of PTEN in Idiopathic Pulmonary Fibrosis. The American Journal of Pathology, 176, 2626-2637. https://doi.org/10.2353/ajpath.2010.091117

[10] Garneau-Tsodikova, S. and Thannickal, V.J. (2008) Protein Kinase Inhibitors in the Treatment of Pulmonary Fibrosis. Current Medicinal Chemistry, 15, 2632-2640. https://doi.org/10.2174/092986708785908969

[11] Gibbons, M.A., MacKinnon, A.C., Ramachandran, P., Dhaliwal, K., Duffin, R., Phythian-Adams, A.T., van Rooijen, N., Haslett, C., Howie, S.E., Simpson, A.J., Hirani, N., Gauldie, J., Iredale, J.P., Sethi, T. and Forbes, S.J. (2011) Ly6Chi Monocytes Direct Alternatively Activated Profibrotic Macrophage Regulation of Lung Fibrosis. American Journal of Respiratory and Critical Care Medicine, 184, 569-581. https://doi.org/10.1164/rccm.201010-1719OC

[12] Murray, L.A., Rosada, R., Moreira, A.P., Joshi, A., Kramer, M.S., Hesson, D.P., Argentieri, R.L., Mathai, S., Gulati, M., Herzog, E.L. and Hogaboam, C.M. (2010) Serum Amyloid P Therapeutically Attenuates Murine Bleomycin-Induced Pulmonary Fibrosis via Its Effects on Macrophages. PLoS ONE, 5, e9683.

https://doi.org/10.1371/journal.pone.0009683

[13] Reynolds, H.Y. (2005) Lung Inflammation and Fibrosis: An Alveolar Macrophage-Centered Perspective from the 1970s to 1980s. American Journal of Respiratory and Critical Care Medicine, 171, 98-102. https://doi.org/10.1164/rccm.200406-788PP

[14] Stahl, M., Schupp, J., Jager, B., Schmid, M., Zissel, G., Muller-Quernheim, J. and Prasse, A. (2013) Lung Collagens Perpetuate Pulmonary Fibrosis via CD204 and M2 Macrophage Activation. PLoS ONE, 8, e81382. https://doi.org/10.1371/journal.pone.0081382

[15] Ho, V.W. and Sly, L.M. (2009) Derivation and Characterization of Murine Alternatively Activated (M2) Macrophages. Methods in Molecular Biology, 531, 173-185.

[16] Ji, R.C. (2012) Macrophages Are Important Mediators of Either Tumor- or Inflammation-Induced Lymphangiogenesis. Cellular and Molecular Life Sciences, 69, 897-914. https://doi.org/10.1007/s00018-011-0848-6

[17] Tabas, I. (2010) Macrophage Death and Defective Inflammation Resolution in Atherosclerosis. Nature Reviews Immunology, 10, 36-46. https://doi.org/10.1038/nri2675

[18] Gordon, S. (2003) Alternative Activation of Macrophages. Nature Reviews Immunology, 3, 23-35. https://doi.org/10.1038/nri978

[19] King Jr., T.E., Pardo, A. and Selman, M. (2011) Idiopathic Pulmonary Fibrosis. The Lancet, 378, 1949-1961. https://doi.org/10.1016/S0140-6736(11)60052-4

[20] Prasse, A., Pechkovsky, D.V., Toews, G.B., Jungraithmayr, W., Kollert, F., Goldmann, T., Vollmer, E., Muller-Quernheim, J. and Zissel, G. (2006) A Vicious Circle of Alveolar Macrophages and Fibroblasts Perpetuates Pulmonary Fibrosis via CCL18. American Journal of Respiratory and Critical Care Medicine, 173, 781-792. https://doi.org/10.1164/rccm.200509-15180C

[21] Tomasek, J.J., Gabbiani, G., Hinz, B., Chaponnier, C. and Brown, R.A. (2002) Myofibroblasts and Mechano-Regulation of Connective Tissue Remodelling. Nature Reviews Molecular Cell Biology, 3, 349-363. https://doi.org/10.1038/nrm809 
[22] Hinz, B., Phan, S.H., Thannickal, V.J., Prunotto, M., Desmouliere, A., Varga, J., De Wever, O., Mareel, M. and Gabbiani, G. (2012) Recent Developments in Myofibroblast Biology: Paradigms for Connective Tissue Remodeling. The American Journal of Pathology, 180, 1340-1355. https://doi.org/10.1016/j.ajpath.2012.02.004

[23] Conte, E., Fruciano, M., Fagone, E., Gili, E., Caraci, F., Iemmolo, M., Crimi, N. and Vancheri, C. (2011) Inhibition of PI3K Prevents the Proliferation and Differentiation of Human Lung Fibroblasts into Myofibroblasts: The Role of Class I P110 Isoforms. PLoS ONE, 6, e24663. https://doi.org/10.1371/journal.pone.0024663

[24] Strieter, R.M., Keeley, E.C., Hughes, M.A., Burdick, M.D. and Mehrad, B. (2009) The Role of Circulating Mesenchymal Progenitor Cells (Fibrocytes) in the Pathogenesis of Pulmonary Fibrosis. Journal of Leukocyte Biology, 86, 1111-1118. https://doi.org/10.1189/jlb.0309132

[25] Ebihara, Y., Masuya, M., Larue, A.C., Fleming, P.A., Visconti, R.P., Minamiguchi, H., Drake, C.J. and Ogawa, M. (2006) Hematopoietic Origins of Fibroblasts: II. In Vitro Studies of Fibroblasts, CFU-F, and Fibrocytes. Experimental Hematology, 34, 219-229. https://doi.org/10.1016/j.exphem.2005.10.008

[26] Bianchetti, L., Barczyk, M., Cardoso, J., Schmidt, M., Bellini, A. and Mattoli, S. (2012) Extracellular Matrix Remodelling Properties of Human Fibrocytes. Journal of Cellular and Molecular Medicine, 16, 483-495. https://doi.org/10.1111/j.1582-4934.2011.01344.x

[27] Hong, K.M., Belperio, J.A., Keane, M.P., Burdick, M.D. and Strieter, R.M. (2007) Differentiation of Human Circulating Fibrocytes as Mediated by Transforming Growth Factor-Beta and Peroxisome Proliferator-Activated Receptor Gamma. The Journal of Biological Chemistry, 282, 22910-22920. https://doi.org/10.1074/jbc.M703597200

[28] Strieter, R.M. and Mehrad, B. (2009) New Mechanisms of Pulmonary Fibrosis. Chest, 136, 1364-1370. https://doi.org/10.1378/chest.09-0510

[29] Araya, J., Kojima, J., Takasaka, N., Ito, S., Fujii, S., Hara, H., Yanagisawa, H., Kobayashi, K., Tsurushige, C., Kawaishi, M., Kamiya, N., Hirano, J., Odaka, M., Morikawa, T., Nishimura, S.L., Kawabata, Y., Hano, H., Nakayama, K. and Kuwano, K. (2013) Insufficient Autophagy in Idiopathic Pulmonary Fibrosis. The American Journal of Physiology Lung Cellular and Molecular Physiology, 304, L56-L69. https://doi.org/10.1152/ajplung.00213.2012

[30] Patel, A.S., Lin, L., Geyer, A., Haspel, J.A., An, C.H., Cao, J., Rosas, I.O. and Morse, D. (2012) Autophagy in Idiopathic Pulmonary Fibrosis. PLoS ONE, 7, e41394. https://doi.org/10.1371/journal.pone.0041394

[31] Ricci, A., Cherubini, E., Scozzi, D., Pietrangeli, V., Tabbi, L., Raffa, S., Leone, L., Visco, V., Torrisi, M.R., Bruno, P., Mancini, R., Ciliberto, G., Terzano, C. and Mariotta, S. (2013) Decreased Expression of Autophagic Beclin 1 Protein in Idiopathic Pulmonary Fibrosis Fibroblasts. Journal of Cellular Physiology, 228, 1516-1524. https://doi.org/10.1002/jcp.24307

[32] Levine, B., Mizushima, N. and Virgin, H.W. (2011) Autophagy in Immunity and Inflammation. Nature, 469, 323-335. https://doi.org/10.1038/nature09782

[33] Mizushima, N., Ohsumi, Y. and Yoshimori, T. (2002) Autophagosome Formation in Mammalian Cells. Cell Structure and Function, 27, 421-429.

[34] Kundu, M. and Thompson, C.B. (2008) Autophagy: Basic Principles and Relevance to Disease. Annual Review of Pathology: Mechanisms of Disease, 3, 427-455. https://doi.org/10.1146/annurev.pathmechdis.2.010506.091842

[35] Pattingre, S., Tassa, A., Qu, X., Garuti, R., Liang, X.H., Mizushima, N., Packer, M., 
Schneider, M.D. and Levine, B. (2005) Bcl-2 Antiapoptotic Proteins Inhibit Beclin 1-Dependent Autophagy. Cell, 122, 927-939. https://doi.org/10.1016/j.cell.2005.07.002

[36] Nho, R.S. and Hergert, P. (2014) IPF Fibroblasts Are Desensitized to Type I Collagen Matrix-Induced Cell Death by Suppressing Low Autophagy via Aberrant Akt/mTOR Kinases. PLoS ONE, 9, e94616. https://doi.org/10.1371/journal.pone.0094616

[37] Xia, H., Diebold, D., Nho, R., Perlman, D., Kleidon, J., Kahm, J., Avdulov, S., Peterson, M., Nerva, J., Bitterman, P. and Henke, C. (2008) Pathological Integrin Signaling Enhances Proliferation of Primary Lung Fibroblasts from Patients with Idiopathic Pulmonary Fibrosis. The Journal of Experimental Medicine, 205, 1659-1672. https://doi.org/10.1084/jem.20080001

[38] Baran, C.P., Opalek, J.M., McMaken, S., Newland, C.A., O’Brien Jr., J.M., Hunter, M.G., Bringardner, B.D., Monick, M.M., Brigstock, D.R., Stromberg, P.C., Hunninghake, G.W. and Marsh, C.B. (2007) Important Roles for Macrophage Colony-Stimulating Factor, CC Chemokine Ligand 2, and Mononuclear Phagocytes in the Pathogenesis of Pulmonary Fibrosis. American Journal of Respiratory and Critical Care Medicine, 176, 78-89. https://doi.org/10.1164/rccm.200609-1279OC

[39] Ganesan, L.P., Wei, G., Pengal, R.A., Moldovan, L., Moldovan, N., Ostrowski, M.C. and Tridandapani, S. (2004) The Serine/Threonine Kinase Akt Promotes Fc Gamma Receptor-Mediated Phagocytosis in Murine Macrophages through the Activation of p70S6 Kinase. The Journal of Biological Chemistry, 279, 54416-54425. https://doi.org/10.1074/jbc.M408188200

[40] Wang, Y., Zeigler, M.M., Lam, G.K., Hunter, M.G., Eubank, T.D., Khramtsov, V.V., Tridandapani, S., Sen, C.K. and Marsh, C.B. (2007) The Role of the NADPH Oxidase Complex, p38 MAPK, and Akt in Regulating Human Monocyte/Macrophage Survival. American Journal of Respiratory Cell and Molecular Biology, 36, 68-77. https://doi.org/10.1165/rcmb.2006-0165OC

[41] Wang, Y., Wang, H., Piper, M.G., McMaken, S., Mo, X., Opalek, J., Schmidt, A.M. and Marsh, C.B. (2010) sRAGE Induces Human Monocyte Survival and Differentiation. The Journal of Immunology, 185, 1822-1835.

https://doi.org/10.4049/jimmunol.0903398

[42] Crawford, J.R., Pilling, D. and Gomer, R.H. (2010) Improved Serum-Free Culture Conditions for Spleen-Derived Murine Fibrocytes. Journal of Immunological Methods, 363, 9-20. https://doi.org/10.1016/j.jim.2010.09.025

[43] Kuroda, E., Ho, V., Ruschmann, J., Antignano, F., Hamilton, M., Rauh, M.J., Antov, A., Flavell, R.A., Sly, L.M. and Krystal, G. (2009) SHIP Represses the Generation of IL-3-Induced M2 Macrophages by Inhibiting IL-4 Production from Basophils. The Journal of Immunology, 183, 3652-3660. https://doi.org/10.4049/jimmunol.0900864

[44] Rauh, M.J., Ho, V., Pereira, C., Sham, A., Sly, L.M., Lam, V., Huxham, L., Minchinton, A.I., Mui, A. and Krystal, G. (2005) SHIP Represses the Generation of Alternatively Activated Macrophages. Immunity, 23, 361-374.

https://doi.org/10.1016/j.immuni.2005.09.003

[45] Phan, S.H., Zhang, K., Zhang, H.Y. and Gharaee-Kermani, M. (1999) The Myofibroblast as an Inflammatory Cell in Pulmonary Fibrosis. Current Topics in Pathology, 93, 173-182.

[46] Hinz, B. and Gabbiani, G. (2010) Fibrosis: Recent Advances in Myofibroblast Biology and New Therapeutic Perspectives. F1000 Biology Reports, 2, 78. https://doi.org/10.3410/B2-78 
[47] Jung, C.H., Ro, S.H., Cao, J., Otto, N.M. and Kim, D.H. (2010) mTOR Regulation of Autophagy. FEBS Letters, 584, 1287-1295.

https://doi.org/10.1016/j.febslet.2010.01.017

[48] Park, H.J., Lee, S.J., Kim, S.H., Han, J., Bae, J., Kim, S.J., Park, C.G. and Chun, T. (2011) IL-10 Inhibits the Starvation Induced Autophagy in Macrophages via Class I Phosphatidylinositol 3-Kinase (PI3K) Pathway. Molecular Immunology, 48, 720-727. https://doi.org/10.1016/j.molimm.2010.10.020

[49] Zhai, C., Cheng, J., Mujahid, H., Wang, H., Kong, J., Yin, Y., Li, J., Zhang, Y., Ji, X. and Chen W. (2014) Selective Inhibition of PI3K/Akt/mTOR Signaling Pathway Regulates Autophagy of Macrophage and Vulnerability of Atherosclerotic Plaque. PLOS ONE, 9, e90563. https://doi.org/10.1371/journal.pone.0090563

[50] Tanida, I., Ueno, T. and Kominami, E. (2004) Human Light Chain 3/MAP1LC3B Is Cleaved at Its Carboxyl-Terminal Met121 to Expose Gly120 for Lipidation and Targeting to Autophagosomal Membranes. The Journal of Biological Chemistry, 279, 47704-47710. https://doi.org/10.1074/jbc.M407016200

[51] Kabeya, Y., Mizushima, N., Ueno, T., Yamamoto, A., Kirisako, T., Noda, T., Kominami, E., Ohsumi, Y. and Yoshimori, T. (2000) LC3, a Mammalian Homologue of Yeast Apg8p, Is Localized in Autophagosome Membranes after Processing. The EMBO Journal, 19, 5720-5728. https://doi.org/10.1093/emboj/19.21.5720

[52] Rogov, V., Dotsch, V., Johansen, T. and Kirkin, V. (2014) Interactions between Autophagy Receptors and Ubiquitin-Like Proteins form the Molecular Basis for Selective Autophagy. Molecular Cell, 53, 167-178. https://doi.org/10.1016/j.molcel.2013.12.014

[53] Puissant, A., Fenouille, N. and Auberger, P. (2012) When Autophagy Meets Cancer through p62/SQSTM1. American Journal of Cancer Research, 2, 397-413.

[54] Hermiston, M.L., Xu, Z. and Weiss, A. (2003) CD45: A Critical Regulator of Signaling Thresholds in Immune Cells. Annual Review of Immunology, 21, 107-137. https://doi.org/10.1146/annurev.immunol.21.120601.140946

[55] Pechkovsky, D.V., Prasse, A., Kollert, F., Engel, K.M., Dentler, J., Luttmann, W., Friedrich, K., Muller-Quernheim, J. and Zissel, G. (2010) Alternatively Activated Alveolar Macrophages in Pulmonary Fibrosis-Mediator Production and Intracellular Signal Transduction. Clinical Immunology, 137, 89-101. https://doi.org/10.1016/j.clim.2010.06.017

[56] White, E.S., Atrasz, R.G., Hu, B., Phan, S.H., Stambolic, V., Mak, T.W., Hogaboam, C.M., Flaherty, K.R., Martinez, F.J., Kontos, C.D. and Toews, G.B. (2006) Negative Regulation of Myofibroblast Differentiation by PTEN (Phosphatase and Tensin Homolog Deleted on Chromosome 10). American Journal of Respiratory and Critical Care Medicine, 173, 112. https://doi.org/10.1164/rccm.200507-1058OC

[57] Wilson, M.S. and Wynn, T.A. (2009) Pulmonary Fibrosis: Pathogenesis, Etiology and Regulation. Mucosal Immunology, 2, 103-121. https://doi.org/10.1038/mi.2008.85

[58] Kleaveland, K.R., Velikoff, M., Yang, J., Agarwal, M., Rippe, R.A., Moore, B.B. and Kim, K.K. (2014) Fibrocytes Are Not an Essential Source of Type I Collagen during Lung Fibrosis. The Journal of Immunology, 193, 5229-5239. https://doi.org/10.4049/jimmunol.1400753

[59] Cha, S.I., Groshong, S.D., Frankel, S.K., Edelman, B.L., Cosgrove, G.P., Terry-Powers, J.L., Remigio, L.K., Curran-Everett, D., Brown, K.K., Cool, C.D. and Riches, D.W. (2010) Compartmentalized Expression of c-FLIP in Lung Tissues of Patients with Idiopathic Pulmonary Fibrosis. American Journal of Respiratory Cell and Molecular Biology, 42, 140-148. https://doi.org/10.1165/rcmb.2008-0419OC 


\section{Supplemental Material}

Supplemental Table 1. qRT-PCR primers.

\begin{tabular}{|c|c|c|}
\hline \multicolumn{3}{|c|}{ Murine primers for qRT-PCR: (5' to $\left.3^{\prime}\right)$} \\
\hline Il-12 & (forward) & ggaagcacggcagcagaata \\
\hline & (reverse) & aacttgagggagaagtaggaatgg \\
\hline Inos & (forward) & accctaagagtcaccaaaatggc \\
\hline & (reverse) & ttgatcctcacatactgtggacg \\
\hline Il-10) & (forward) & gccaagccttatcggaaatg \\
\hline & (reverse) & tttctgggccatgcttctct \\
\hline Ym1 & (forward) & ctggaattggtgcccctaca \\
\hline & (reverse) & caagcatggtggttttacagga \\
\hline ColIa & (forward) & atggattcccgttcgagtacg \\
\hline & (reverse) & tcagctggatagcgacatcg \\
\hline ColIIIb & (forward) & caccettcttcatcccactctta \\
\hline & (reverse) & accaaggtggctgcatcc \\
\hline aSMA & (forward) & gctcagccagatgcaatcaa \\
\hline & (reverse) & ccttggccacaatggtcttg \\
\hline Beclin1 & (forward) & caatgtcttcaatgccacctt \\
\hline & (reverse) & ttcattccactccacaggaac \\
\hline Map1lc3a & (forward) & gcctgtcctggataagacca \\
\hline & (reverse) & ggttgaccagcaggaagaag \\
\hline Map1lc3b & (forward) & cgtcctggacaagaccaagt \\
\hline & (reverse) & ccattcaccaggaggaagaa \\
\hline
\end{tabular}


(a)

AKT activity in macrophages

in the absence of CSF1

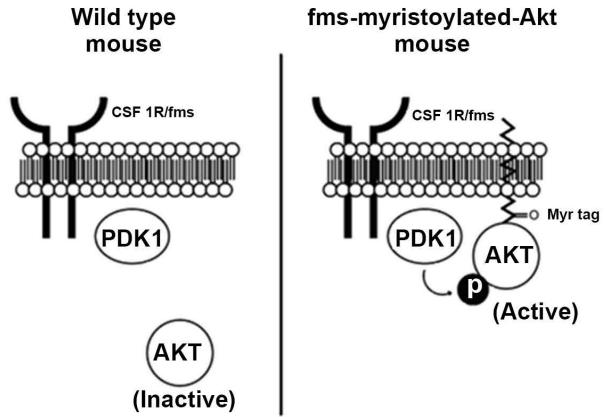

(b)

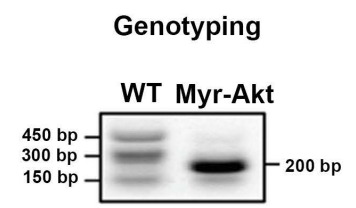

(c)

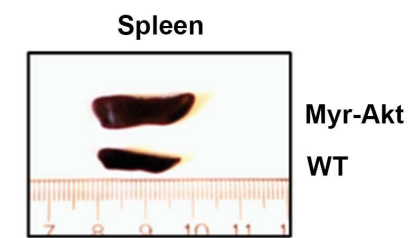

Supplemental Figure 1. Schema for constitutive activation of AKT and identification of Myr-Akt mice. (a) AKT is active in macrophages in the absence of CSF1 in Myr-Akt mice. (b) Genotyping identifies the 200 bp band that distinguishes Myr-Akt from WT mice. (c) Splenomegaly as confirmation for Myr-Akt penetrance. 
(a)

Lung; Hematoxylin \& Eosin (H\&E)

WT

Myr-Akt
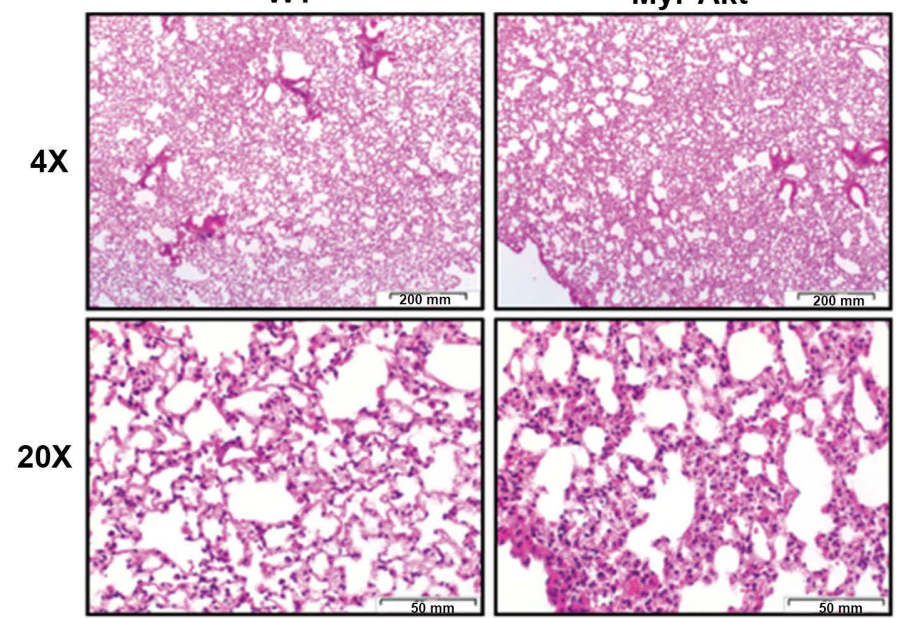

(b)

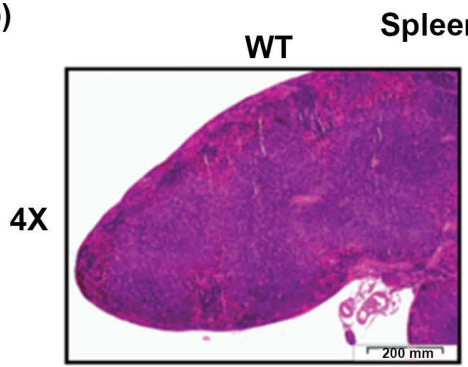

Spleen; H\&E

Myr-Akt
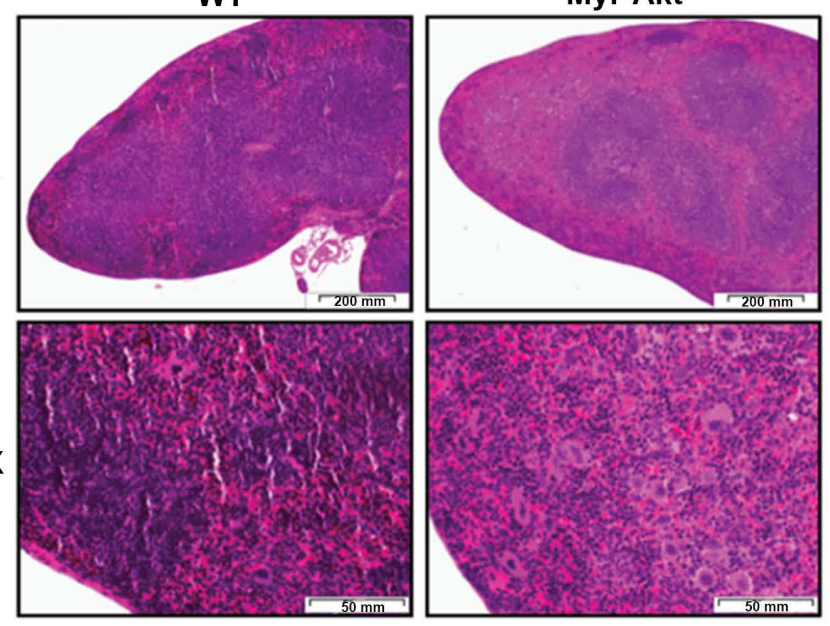

Supplemental Figure 2. Tissue architecture in Myr-Akt mice. 6 8-week-old WT or Myr-Akt mice were sacrificed and (a) lungs and (b) spleens harvested, paraffin embedded, and subjected to Hematoxylin \& Eosin (H\&E) staining and microscopy using $4 \mathrm{X}$ and 20X objectives (40X and $200 \times$ magnification, respectively). 
(a)
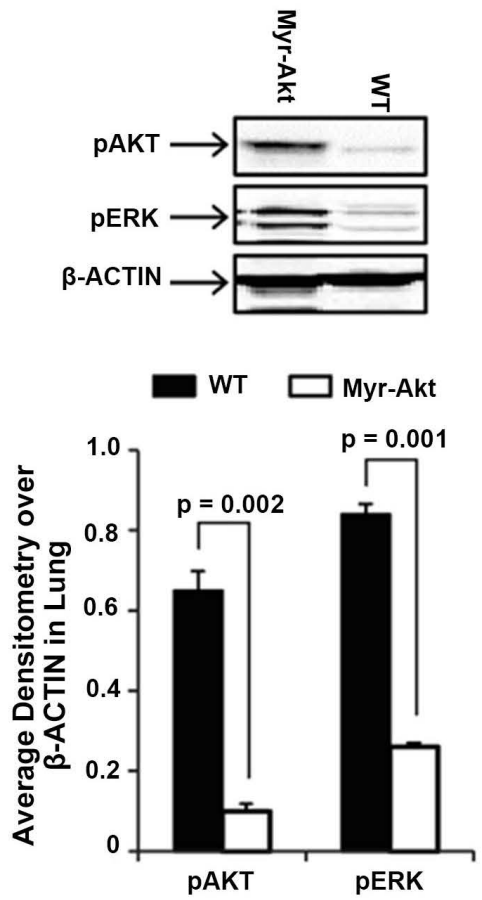

(b)

Spleen
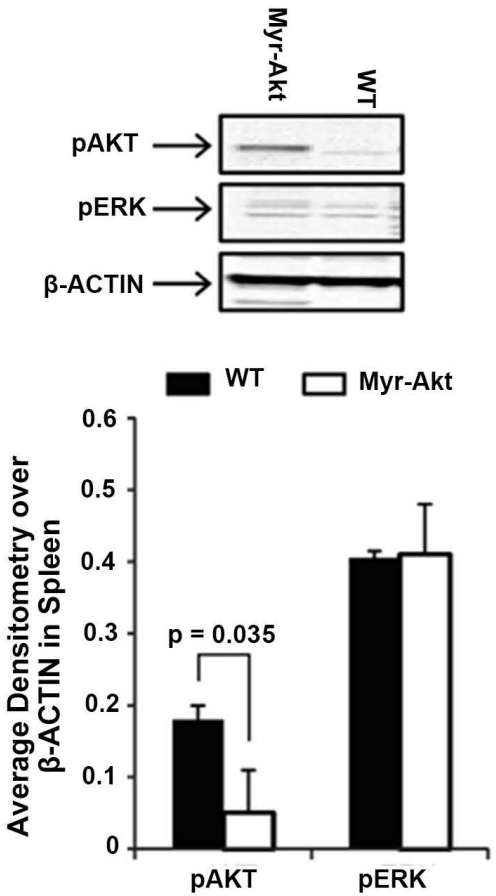

(c)

Bone Marrow
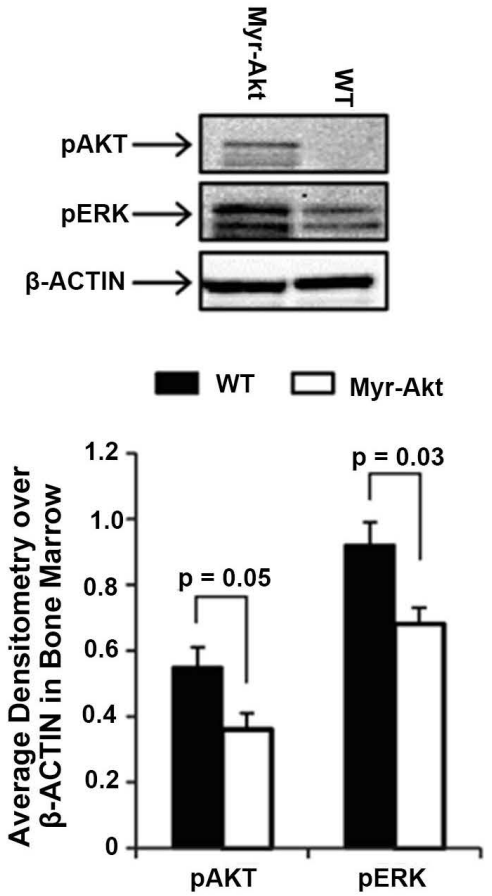

Supplemental Figure 3. Signaling pathway activation in various tissues in Myr-Akt mice. 6 - 8-week-old WT or Myr-Akt mice were sacrificed and (a) lungs, (b) spleens, and (c) bone marrow were harvested and subjected to Western Blot analysis for differences in phosphor-Akt and phospho-EKR relative to $\beta$-ACTIN expression. 